\title{
THE CLOSURE-COMPLEMENT-FRONTIER PROBLEM IN SATURATED POLYTOPOLOGICAL SPACES
}

\author{
Sara Canilang, Michael P. Cohen, Nicolas Graese, and Ian Seong
}

(Received 30 March, 2020)

\begin{abstract}
Let $X$ be a space equipped with $n$ topologies $\tau_{1}, \ldots, \tau_{n}$ which are pairwise comparable and saturated, and for each $1 \leq i \leq n$ let $k_{i}$ and $f_{i}$ be the associated topological closure and frontier operators, respectively. Inspired by the closure-complement theorem of Kuratowski, we prove that the monoid of set operators $\mathcal{K F}_{n}$ generated by $\left\{k_{i}, f_{i}: 1 \leq i \leq n\right\} \cup\{c\}$ (where $c$ denotes the set complement operator) has cardinality no more than $2 p(n)$ where $p(n)=$ $\frac{5}{24} n^{4}+\frac{37}{12} n^{3}+\frac{79}{24} n^{2}+\frac{101}{12} n+2$. The bound is sharp in the following sense: for each $n$ there exists a saturated polytopological space $\left(X, \tau_{1}, \ldots, \tau_{n}\right)$ and a subset $A \subseteq X$ such that repeated application of the operators $k_{i}, f_{i}, c$ to $A$ will yield exactly $2 p(n)$ distinct sets. In particular, following the tradition for Kuratowski-type problems, we exhibit an explicit initial set in $\mathbb{R}$, equipped with the usual and Sorgenfrey topologies, which yields $2 p(2)=120$ distinct sets under the action of the monoid $\mathcal{K F}_{2}$.
\end{abstract}

\section{Introduction}

In his 1922 thesis $\mathbf{8}$, Kuratowski posed and solved the following problem: given a topological space $(X, \tau)$, what is the largest number of distinct subsets that can be obtained by starting from an initial set $A \subseteq X$, and applying the topological closure and complement operators, in any order, as often as desired? The answer is 14. This result, now widely known as Kuratowski's closure-complement theorem, is both thought-provoking and amusing, and has inspired a substantial number of authors to study generalizations, variants, and elaborations of the original closurecomplement problem. We recommend consulting the admirable survey of Gardner and Jackson [6], or visiting Bowron's website Kuratowski's Closure-Complement Cornucopia [3] for an indexed list of all relevant literature.

Shallit and Willard 10 considered a natural extension of Kuratowski's problem. If we equip a space $X$ with not one but two distinct topologies $\tau_{1}$ and $\tau_{2}$, how many distinct subsets may be obtained by starting with an initial set, and applying each of the two associated closure operators $k_{1}, k_{2}$, and the set complement operator $c$, in any order, as often as desired? The authors construct an example of a bitopological space $\left(X, \tau_{1}, \tau_{2}\right)$ where it is possible to obtain infinitely many subsets from a certain initial set. Consequently, the monoid $\mathcal{K}_{2}$ of set operators generated by $\left\{k_{1}, k_{2}, c\right\}$ may have infinitely many elements in general. In their example, the topologies $\tau_{1}$ and $\tau_{2}$ are incomparable, which suggests that the monoid may yet be finite in case $\tau_{1} \supseteq \tau_{2}$.

In [1], Banakh, Chervak, Martynyuk, Pylypovych, Ravsky, and Simkiv verify this last possibility, and generalize the closure-complement theorem to polytopological

2010 Mathematics Subject Classification 54A10, 54E55, 06F05. 
spaces, i.e. sets $X$ equipped with families of topologies $\mathcal{T}$ in which the topologies are linearly ordered by inclusion. If the family is a finite set $\mathcal{T}=\left\{\tau_{1}, \ldots, \tau_{n}\right\}$, they give an explicit formula for the maximal cardinality of the monoid $\mathcal{K}_{n}$ generated by $\left\{k_{j}: 1 \leq j \leq n\right\} \cup\{c\}$. This maximal cardinality is of course 14 when $n=1$, and grows exponentially as $n \rightarrow \infty$.

The authors of [1] also consider the special case where the topologies involved are saturated, i.e., for any $1 \leq j, \ell \leq n$, if a nonempty set $U$ is $\tau_{j}$-open, then $U$ has nonempty $\tau_{\ell}$-interior. In the saturated case, the cardinality bound on the monoid is given by $\# \mathcal{K}_{n} \leq 12 n+2$. The most natural example is the case of the real line $\mathbb{R}$ equipped with $\tau_{2}=$ the usual topology and $\tau_{1}=$ the Sorgenfrey topology. Then one may obtain no more than $12 \cdot 2+2=26$ distinct sets by applying $k_{1}, k_{2}, c$ to any particular initial set, and indeed this upper bound is obtainable in $\left(\mathbb{R}, \tau_{1}, \tau_{2}\right)$, as demonstrated explicitly in [1].

In [5, Gaida and Eremenko solved a closure-complement-frontier problem by showing that in any topological space $(X, \tau)$, the monoid $\mathcal{K F}_{1}$ generated by $\{k, f, c\}$ (where $f$ is the frontier operator, or topological boundary operator) has cardinality $\leq 34$; moreover there are examples of spaces in which it is possible to obtain 34 distinct subsets by applying the operators to a single initial set. This problem also appeared as Problem E3144 in American Mathematical Monthly [2]. The purpose of this paper is to study the extension of Gaida and Eremenko's problem to the setting of saturated polytopological spaces as in [1.

To state our result, we consider a polytopological space $\left(X, \tau_{1}, \ldots, \tau_{n}\right)$, and we denote by $\mathcal{K F}_{n}=\mathcal{K F}_{n}\left(X, \tau_{1}, \ldots, \tau_{n}\right)$ the monoid of set operators generated by $\left\{k_{j}, f_{j}: 1 \leq j \leq n\right\} \cup\{c\}$. We also let $\mathcal{K F}_{n}^{0}=\mathcal{K} \mathcal{F}_{n}^{0}\left(X, \tau_{1}, \ldots, \tau_{n}\right)$ denote the monoid generated by $\left\{k_{j}, i_{j}, f_{j}: 1 \leq j \leq n\right\}$, where $i_{j}$ is the interior operator associated to $\tau_{j}$. Since $i_{j}=c k_{j} c$, we have that $\mathcal{K F}_{n}^{0} \subseteq \mathcal{K} \mathcal{F}_{n}$, and in fact, in Section 2 we observe that

$$
\mathcal{K} \mathcal{F}_{n}=\mathcal{K} \mathcal{F}_{n}^{0} \cup c \mathcal{K} \mathcal{F}_{n}^{0}
$$

so that $\mathcal{K F}_{n}^{0}$ comprises the submonoid of even operators of $\mathcal{K} \mathcal{F}_{n}$, and

$$
\# \mathcal{K} \mathcal{F}_{n}=2 \cdot \# \mathcal{K} \mathcal{F}_{n}^{0}
$$

Our main theorem follows.

Theorem 1.1. Let $\left(X, \tau_{1}, \ldots, \tau_{n}\right)$ be a saturated polytopological space. Then $\# \mathcal{K F}_{n}^{0} \leq$ $p(n)$ and $\# \mathcal{K} \mathcal{F}_{n}=2 \cdot \# \mathcal{K} \mathcal{F}_{n}^{0} \leq 2 p(n)$, where

$$
p(n)=\frac{5}{24} n^{4}+\frac{37}{12} n^{3}+\frac{79}{24} n^{2}+\frac{101}{12} n+2 .
$$

Thus for $n=1$ we recover Gaida-Eremenko's result with $p(n)=17$ and $2 p(n)=$ 34. The next few upper bounds are $p(2)=60, p(3)=157, p(4)=339$, and $p(5)=642$.

We also demonstrate that the bound $p(n)$ is sharp.

Theorem 1.2. For every $n \geq 1$, there exists a saturated polytopological space $\left(X, \tau_{1}, \ldots, \tau_{n}\right)$ in which $\# \mathcal{K} \mathcal{F}_{n}^{0}=p(n)$ and $\# \mathcal{K} \mathcal{F}_{n}=2 p(n)$. In fact, there is an initial set $A \subseteq X$ such that $\#\left\{o A: o \in \mathcal{K F}_{n}\right\}=2 p(n)$. 
The explicit examples we give are natural and easy to understand (disjoint unions of copies of $\mathbb{R}$ equipped with combinations of the Sorgenfrey and Euclidean topologies), but not finite. By the results of [9] (see [6] Theorem 4.1 and surrounding remarks), we deduce abstractly that there must exist a finite polytopological space $\left(X, \tau_{1}, \ldots, \tau_{n}\right)$ on which $\# \mathcal{K} \mathcal{F}_{n}^{0}=p(n)$, but we do not know how many points are necessary.

Question 1.3. What is the minimal cardinality of a polytopological space $\left(X, \tau_{1}, \ldots, \tau_{n}\right)$ for which $\# \mathcal{K} \mathcal{F}_{n}^{0}=p(n)$ exactly? What is the minimal cardinality of a space in which one can find an initial set $A$ with $\#\left\{o A: o \in \mathcal{K F}_{n}\right\}=2 p(n)$ ?

It would be interesting to know the answer even for $n=2$. It is known that the minimal number of points needed for a space to contain a Kuratowski 14-set is 7; see [7. During the preparation of this article, Bowron has communicated to us that if $n=1$, then the minimal number of points needed for $\# \mathcal{K} \mathcal{F}_{1}^{0}=17$ is four, while the minimal number of points needed to contain a 34 -set is 8 .

Another interesting question that remains open is to solve the closure-complementfrontier problem for polytopological spaces which are not necessarily saturated.

Question 1.4. Let $\left(X, \tau_{1}, \ldots, \tau_{n}\right)$ be a polytopological space which is not necessarily saturated. What is the maximal cardinality of the monoid $\mathcal{K} \mathcal{F}_{n}$ generated by $\left\{k_{j}, f_{j}: 1 \leq j \leq n\right\} \cup\{c\} ?$

Finally, it would be interesting to study some of the variants described in Section 4 of $\mathbf{6}$ in the larger context of polytopological spaces. For example, it was shown independently by Gardner and Jackson [6] and by Sherman [11 that in any topological space $(X, \tau)$, the greatest number of sets one may obtain from an initial set $A \subseteq X$ by applying the set operators $\{k, i, \cup, \cap\}$ is 35 .

Question 1.5. Let $\left(X, \tau_{1}, \ldots, \tau_{n}\right)$ be a (saturated?) polytopological space. What is the largest number of sets one may obtain from an initial set $A \subseteq X$ by applying the set operators $k_{j}, i_{j}(1 \leq j \leq n), \cup$, and $\cap$ in any order, as often as desired?

\section{Preliminaries and Notation}

Recall from the introduction that a polytopological space is a set $X$ equipped with a family of topologies $\mathcal{T}$ which is linearly ordered by the inclusion relation. In this paper we will work only with finite families $\mathcal{T}=\left\{\tau_{1}, \ldots, \tau_{n}\right\}$ and assume $\tau_{1} \supseteq \ldots \supseteq \tau_{n}$. In this case we refer to $\left(X, \tau_{1}, \ldots, \tau_{n}\right)$ as an $n$-topological space.

For each topology $\tau_{j}$, we permanently associate the closure operator $k_{j}$, the interior operator $i_{j}$, and the frontier operator $f_{j}$. We use $c$ to denote the set complement operator. The operators $k_{j}$ and $i_{j}$ are idempotent, so $k_{j} k_{j}=k_{j}$ and $i_{j} i_{j}=i_{j}$, and the operator $c$ is an involution, so $c c=\mathrm{Id}$, where Id denotes the identity operator. For each set $A \subseteq X$ we have $f_{j} A=k_{j} A \cap k_{j} c A$; we summarize this symbolically by writing

$$
f_{j}=k_{j} \wedge k_{j} c=k_{j} \wedge c i_{j}
$$

From the identity above, we see that

$$
f_{j} c=f_{j}
$$


We permanently denote by $\mathcal{K F}_{n}=\mathcal{K} \mathcal{F}_{n}\left(X, \tau_{1}, \ldots, \tau_{n}\right)$ the smallest monoid of set operators which contains $k_{j}, f_{j}(1 \leq j \leq n)$ and $c$. We also denote by $\mathcal{K} \mathcal{F}_{n}^{0}=$ $\mathcal{K} \mathcal{F}_{n}^{0}\left(X, \tau_{1}, \ldots, \tau_{n}\right)$ the smallest monoid of set operators which contains $k_{j}, i_{j}$, and $f_{j}(1 \leq j \leq n)$. By DeMorgan's laws, we have $c k_{j} c=i_{j}$ and thus it is immediate that $\mathcal{K F}_{n}^{0} \subseteq \mathcal{K} \mathcal{F}_{n}$.

Since we are requiring that $\mathcal{K F}_{n}^{0}$ be a monoid, it contains the identity operator Id. It also contains the zero operator 0 , i.e. the set operator for which $0 A=\emptyset$, for every $A \subseteq X$. This follows from the work of Gaida and Eremenko [5], who observed that

$$
i_{1} f_{1} k_{1}=0
$$

We also define the one operator by the rule $1=c 0$, so $1 A=X$ for every $A \subseteq X$ and $1 \in \mathcal{K F}_{n}$.

Proposition 2.1. The sets $\mathcal{K F}_{n}^{0}$ and $c \mathcal{K} \mathcal{F}_{n}^{0}$ are disjoint and $\mathcal{K} \mathcal{F}_{n}$ is equal to their union.

Proof. By examining the generators $k_{j}, i_{j}, f_{j}$ of $\mathcal{K F}_{n}^{0}$, it is clear that $o \emptyset=\emptyset$ and $\operatorname{co}=X$ for any operator $o \in \mathcal{K} \mathcal{F}_{n}^{0}$. Therefore, $\mathcal{K F}_{n}^{0}$ and $c \mathcal{K} \mathcal{F}_{n}^{0}$ are disjoint.

To see that $\mathcal{K F}_{n} \subseteq \mathcal{K} \mathcal{F}_{n}^{0} \cup c \mathcal{K} \mathcal{F}_{n}^{0}$, we can argue by induction on word length of elements of $\mathcal{K F}_{n}$. Let $\mathcal{W}_{m} \subseteq \mathcal{K} \mathcal{F}_{n}$ be the set of operators which can be written as a word of length $\leq m$ in the generators $k_{j}, f_{j}, c$. Assume that $\mathcal{W}_{m} \subseteq \mathcal{K} \mathcal{F}_{n}^{0} \cup c \mathcal{K} \mathcal{F}_{n}^{0}$ (which is certainly true if $m=1$ ). Then $\mathcal{W}_{m+1}$ is the union of sets of the form $k_{j} \mathcal{W}_{m}, f_{j} \mathcal{W}_{m}$, and $c \mathcal{W}_{m}$. But by invoking DeMorgan's laws and the identity $f_{j} c=f_{j}$, the inductive hypothesis implies the following inclusions:

$$
\begin{aligned}
k_{j} \mathcal{W}_{m} & \subseteq k_{j} \mathcal{K} \mathcal{F}_{n}^{0} \cup k_{j} c \mathcal{K} \mathcal{F}_{n}^{0} \\
& =k_{j} \mathcal{K} \mathcal{F}_{n}^{0} \cup c i_{j} \mathcal{K} \mathcal{F}_{n}^{0}=\mathcal{K} \mathcal{F}_{n}^{0} \cup c \mathcal{K} \mathcal{F}_{n}^{0} ; \\
f_{j} \mathcal{W}_{m} & \subseteq f_{j} \mathcal{K} \mathcal{F}_{n}^{0} \cup f_{j} c \mathcal{K} \mathcal{F}_{n}^{0} \\
& =f_{j} \mathcal{K} \mathcal{F}_{n}^{0} \cup f_{j} \mathcal{K} \mathcal{F}_{n}^{0}=\mathcal{K} \mathcal{F}_{n}^{0} \\
c \mathcal{W}_{m} & \subseteq c \mathcal{K} \mathcal{F}_{n}^{0} \cup c c \mathcal{K} \mathcal{F}_{n}^{0}=\mathcal{K} \mathcal{F}_{n}^{0} \cup c \mathcal{K} \mathcal{F}_{n}^{0} ;
\end{aligned}
$$

which concludes the inductive step and the proof.

By the previous proposition, we are now justified in referring to the elements of $\mathcal{K F}_{n}^{0}$ as the even operators, and those in $c \mathcal{K} \mathcal{F}_{n}^{0}$ as the odd operators. By direct algebraic manipulation, it is easy to see that any operator in $\mathcal{K} \mathcal{F}_{n}$ may be rewritten as a word in which the generator $c$ appears either zero times (the even case) or exactly one time (the odd case). For example $k_{1} c i_{1} c c k_{1} c f_{1} k_{1} c=k_{1} i_{1} f_{1} i_{1}$.

Corollary 2.2. $\# \mathcal{K} \mathcal{F}_{n}=2 \cdot \# \mathcal{K} \mathcal{F}_{n}^{0}$. 
In the special case $n=1$, the results of Gaida-Eremenko 5 imply that $\mathcal{K} \mathcal{F}_{1}^{0}$ consists of no more than 17 distinct even operators, which may be listed explicitly as below:

$$
\begin{gathered}
\mathcal{K} \mathcal{F}_{1}^{0}=\left\{\operatorname{Id}, k_{1}, i_{1}, k_{1} i_{1}, i_{1} k_{1}, i_{1} k_{1} i_{1}, k_{1} i_{1} k_{1}, f_{1}, f_{1} f_{1}, f_{1} k_{1}, f_{1} i_{1}, i_{1} f_{1},\right. \\
\left.k_{1} i_{1} f_{1}, 0, f_{1} k_{1} i_{1}, f_{1} i_{1} k_{1}, f_{1} i_{1} f_{1}\right\} .
\end{gathered}
$$

Adding $c$ to the left of each operator above yields the odd operators, for a total of $\# \mathcal{K} \mathcal{F}_{n} \leq 34$. The operators are indeed distinct when, for instance, $X=\mathbb{R}$ and $\tau_{1}$ is the usual topology on the reals, and in this case we get $\# \mathcal{K} \mathcal{F}_{n}=34$.

We are ready to state some elementary algebraic identities in $\mathcal{K F}_{n}^{0}$, which are easily proven. The first one is prominent in the solution to Kuratowski's original closure-complement problem.

Lemma 2.3. In any $n$-topological space $\left(X, \tau_{1}, \ldots, \tau_{n}\right)$,

(1) (Kuratowski) for each $1 \leq x \leq n$,

(2) for each $1 \leq x, y \leq n$,

$$
k_{x} i_{x} k_{x} i_{x}=k_{x} i_{x} \text { and } i_{x} k_{x} i_{x} k_{x}=i_{x} k_{x}
$$

(3) for each $1 \leq x, y \leq n$,

$$
k_{x} k_{y}=k_{\max (x, y)} \text { and } i_{x} i_{y}=i_{\max (x, y)}
$$

$$
\text { if } x \leq y \text { then } k_{x} f_{y}=f_{y} .
$$

Recall that an $n$-topological space $\left(X, \tau_{1}, \ldots, \tau_{n}\right)$ is saturated if whenever $1 \leq$ $x, y \leq n$ and $U$ is a nonempty $\tau_{x}$-open set, then $i_{y} U \neq \emptyset$. For the remainder of the paper, we assume that our space $\left(X, \tau_{1}, \ldots, \tau_{n}\right)$ is saturated. The most basic and important identity, which we use extensively, is proven in [1]:

Lemma 2.4 (Banakh, Chervak, Martynyuk, Pylypovych, Ravsky, Simkiv). Let $\left(X, \tau_{1}, \ldots, \tau_{n}\right)$ be a saturated $n$-topological space. For each $1 \leq x, y \leq n, k_{x} i_{y}=k_{x} i_{x}$ and $i_{x} k_{y}=i_{x} k_{x}$.

This identity means that, assuming saturation, the second index in a word of the form $k_{x} i_{y}$ or $i_{x} k_{y}$ is irrelevant in determining the action of the operator. For this reason, we find it convenient to adopt a star notation, and simply write

$$
\text { for each } 1 \leq x, y \leq n, k_{x} i_{y}=k_{x} i_{*} \text { and } i_{x} k_{y}=i_{x} k_{*} \text {. }
$$

We employ this notation in the following lemma.

Lemma 2.5 (IF Lemma). Let $\left(X, \tau_{1}, \ldots, \tau_{n}\right)$ be a saturated $n$-topological space. For each $1 \leq x, y \leq n$,

$$
i_{x} f_{y}=i_{x} f_{*} .
$$

Proof. Since interiors distribute over intersections, by Lemma 2.4 we have $i_{x} f_{y}=$ $i_{x} k_{y} \wedge i_{x} k_{y} c=i_{x} k_{*} \wedge i_{x} k_{*} c=i_{x} f_{*}$.

For other types of words, as below, it turns out that the value of $y$ is irrelevant if $y \leq x$, but may matter if $y>x$. 
Lemma 2.6 (FK Lemma). Let $\left(X, \tau_{1}, \ldots, \tau_{n}\right)$ be a saturated $n$-topological space. For each $1 \leq x, y \leq n$,

$$
f_{x} k_{y}=f_{x} k_{\max (x, y)}
$$

Proof. If $y \geq x$ then the statement is trivial. Otherwise $y<x$, and we compute using Lemmas 2.3 and 2.4 that $f_{x} k_{y}=k_{x} k_{y} \wedge c i_{x} k_{y}=k_{x} \wedge c i_{x} k_{*}=f_{x} k_{x}=$ $f_{x} k_{\max (x, y)}$.

For many of our algebraic lemmas involving $k_{x}$ or $i_{x}$, we may use DeMorgan's law to instantly deduce a "dual" corollary.

Lemma 2.7 (FI Lemma). Let $\left(X, \tau_{1}, \ldots, \tau_{n}\right)$ be a saturated $n$-topological space. For each $1 \leq x, y \leq n$,

$$
f_{x} i_{y}=f_{x} i_{\max (x, y)}
$$

Proof. By duality: $f_{x} i_{y}=f_{x} c k_{y} c=f_{x} k_{y} c=f_{x} k_{\max (x, y)} c=f_{x} c i_{\max (x, y)}=$ $f_{x} i_{\max (x, y)}$.

Lemma 2.8 (FKF Lemma). Let $\left(X, \tau_{1}, \ldots, \tau_{n}\right)$ be a saturated $n$-topological space. Then for each $1 \leq x, y, z \leq n$,

$$
\text { if } y \leq \max (x, z) \text {, then } f_{x} k_{y} f_{z}=f_{x} f_{z} \text {. }
$$

Proof. If $y \leq z$ then $k_{y} f_{z}=f_{z}$ by Lemma 2.3. Otherwise $y \leq x$, in which case we compute

$$
\begin{aligned}
f_{x} k_{y} f_{z} & =k_{x} k_{y} f_{z} \wedge c i_{x} k_{y} f_{z} \\
& =k_{x} f_{z} \wedge c i_{x} k_{*} f_{z} \\
& =k_{x} f_{z} \wedge c i_{x} f_{z}=f_{x} f_{z} .
\end{aligned}
$$

Lemma 2.9 (FIKI/FKIK/FKIF Lemma). Let $\left(X, \tau_{1}, \ldots, \tau_{n}\right)$ be a saturated $n$ topological space. For each $1 \leq x, y \leq n$,

- if $y \leq x$, then $f_{x} i_{y} k_{*} i_{*}=f_{x} k_{x} i_{*}$.

- if $y \leq x$, then $f_{x} k_{y} i_{*} k_{*}=f_{x} i_{x} k_{*}$.

- if $y \leq x$, then $f_{x} k_{y} i_{*} f_{*}=f_{x} i_{x} f_{*}$.

Proof. For the first item, by Lemmas 2.3 and 2.4 . compute

$$
\begin{aligned}
f_{x} i_{y} k_{*} i_{*} & =k_{x} i_{*} k_{*} i_{*} \wedge k_{x} c i_{y} k_{*} i_{*} \\
& =k_{x} i_{*} \wedge c i_{x} i_{y} k_{*} i_{*} \\
& =k_{x} k_{x} i_{*} \wedge c i_{x} k_{x} i_{*} \\
& =f_{x} k_{x} i_{*} .
\end{aligned}
$$


The second item follows from the first by duality. The third follows from the second, by observing that $f_{x} k_{y} i_{*} f_{*}=f_{x} k_{y} i_{*} k_{*} f_{*}=f_{x} i_{x} k_{*} f_{*}=f_{x} i_{x} f_{*}$.

The next lemma is a generalization of Gaida-Eremenko's observation, together with its dual statement.

Lemma 2.10 (IFK/IFI Lemma). Let $\left(X, \tau_{1}, \ldots, \tau_{n}\right)$ be a saturated $n$-topological space.

- For any $1 \leq x, y, z \leq n, i_{x} f_{y} k_{z}=0$.

- For any $1 \leq x, y, z \leq n, i_{x} f_{y} i_{z}=0$.

Proof. It suffices to prove that $i_{n} f_{y} k_{z}=0$, for if there existed a set $A \subseteq X$ with $i_{x} f_{y} k_{z} A \neq \emptyset$, then by saturation, we would have $i_{n} f_{y} k_{z} A=i_{n} i_{x} f_{y} k_{z} A \neq \emptyset$, which would contradict $i_{n} f_{y} k_{z}=0$.

We can use Lemma 2.5 to rewrite $i_{n} f_{y} k_{z}=i_{n} f_{*} k_{z}=i_{n} f_{n} k_{z}$. Then use Lemma 2.6 to write $i_{n} f_{y} k_{z}=i_{n} f_{n} k_{n}=0$.

Lemma 2.11 (FFK/FFI/FFF Lemma). Let $\left(X, \tau_{1}, \ldots, \tau_{n}\right)$ be a saturated $n$-topological space. For each $1 \leq x, y, z \leq n$, the following hold.

- $f_{x} f_{y} k_{z}=k_{x} f_{y} k_{z}$.

- If $x \leq y$, then $f_{x} f_{y} k_{z}=f_{y} k_{z}$.

- $f_{x} f_{y} i_{z}=k_{x} f_{y} i_{z}$.

- If $x \leq y$, then $f_{x} f_{y} i_{z}=f_{y} i_{z}$.

- $f_{x} f_{y} f_{z}=k_{x} f_{y} f_{z}$.

- If $x \leq y$, then $f_{x} f_{y} f_{z}=f_{y} f_{z}$.

Proof. It suffices to prove the first statement, as the second follows immediately; the third and fourth follow from duality; and the fifth and sixth follow from the observation that $f_{x} f_{y} f_{z}=f_{x} f_{y} k_{z} f_{z}$.

Using Lemma 2.10, we compute

$$
\begin{aligned}
f_{x} f_{y} k_{z} & =k_{x} f_{y} k_{z} \wedge k_{x} c f_{y} k_{z} \\
& =k_{x} f_{y} k_{z} \wedge c i_{x} f_{y} k_{z} \\
& =k_{x} f_{y} k_{z} \wedge c 0 \\
& =k_{x} f_{y} k_{z} \wedge 1=k_{x} f_{y} k_{z} .
\end{aligned}
$$

Lemma 2.12 (FKFK/FKFI Lemma). Let $\left(X, \tau_{1}, \ldots, \tau_{n}\right)$ be a saturated $n$-topological space.

- For any $1 \leq x, y, z, w \leq n, f_{x} k_{y} f_{z} k_{w}=k_{\max (x, y)} f_{z} k_{w}$.

- For any $1 \leq x, y, z, w \leq n, f_{x} k_{y} f_{z} i_{w}=k_{\max (x, y)} f_{z} i_{w}$. 
Proof. Using Lemma 2.10 again,

$$
\begin{aligned}
f_{x} k_{y} f_{z} k_{w} & =k_{x} k_{y} f_{z} k_{w} \wedge c i_{x} k_{y} f_{z} k_{w} \\
& =k_{\max (x, y)} f_{z} k_{w} \wedge c i_{x} k_{*} f_{z} k_{w} \\
& =k_{\max (x, y)} f_{z} k_{w} \wedge c i_{x} f_{z} k_{w} \\
& =k_{\max (x, y)} f_{z} k_{w} \wedge c 0 \\
& =k_{\max (x, y)} f_{z} k_{w} \wedge 1=k_{\max (x, y)} f_{z} k_{w} .
\end{aligned}
$$

\section{The Case of Two Topologies}

In this section we look closely at the special case where $n=2$, and solve the closure-complement-frontier problem for a saturated 2-topological space. The prototypical example is $\left(\mathbb{R}, \tau_{s}, \tau_{u}\right)$ where $\tau_{s}=$ the Sorgenfrey topology (in which basic open neighborhoods have the form $[a, b)=\{x \in \mathbb{R}: a \leq x<b\})$ and $\tau_{u}=$ the usual Euclidean topology.

It is instructive to use Lemmas 2.3 through 2.12 to write out the distinct elements of $\mathcal{K F}_{2}^{0}$ explicitly. There turn out to be at most 60 of them. This is an enjoyable computation and we postpone the details until the more general case of Section 4 , where $n$ is arbitrary. The reader may verify the truth of the following proposition by observing that applying any of the generators $k_{x}, i_{x}$, or $f_{x}(x=1,2)$ to the left of any of the 60 words listed below will always simply produce another word on the list, and thus the entire monoid $\mathcal{K} \mathcal{F}_{2}^{0}$ is accounted for.

Proposition 3.1. The monoid $\mathcal{K F}_{2}^{0}$ consists of at most 60 elements, which are listed in the table below. Consequently, the monoid $\mathcal{K F}_{2}$ consists of at most 120 elements.

\begin{tabular}{|c|l|c|}
\hline Word Length & Operators & Count \\
\hline 0 & Id & 1 \\
\hline 1 & $i_{1}, i_{2}, \quad k_{1}, k_{2}, \quad f_{1}, f_{2}$ & 6 \\
\hline 2 & $k_{1} i_{*}, k_{2} i_{*}, \quad i_{1} k_{*}, i_{2} k_{*}, \quad f_{1} i_{1}, f_{1} i_{2}, f_{2} i_{2}$, & 17 \\
& $i_{1} f_{*}, i_{2} f_{*}, \quad f_{1} k_{1}, f_{1} k_{2}, f_{2} k_{2}$, & \\
& $k_{2} f_{1}, \quad f_{1} f_{1}, f_{1} f_{2}, f_{2} f_{1}, f_{2} f_{2}$ & \\
\hline 3 & $i_{1} k_{*} i_{*}, i_{2} k_{*} i_{*}, k_{1} i_{*} k_{*}, k_{2} i_{*} k_{*}, f_{1} k_{1} i_{*}, f_{1} k_{2} i_{*}, f_{2} k_{2} i_{*}$, & 23 \\
& $f_{1} i_{1} k_{*}, f_{1} i_{2} k_{*}, f_{2} i_{2} k_{*}, \quad 0, \quad k_{2} f_{1} i_{1}, k_{2} f_{1} i_{2}$, & \\
& $k_{1} i_{*} f_{*}, k_{2} i_{*} f_{*} \quad k_{2} f_{1} k_{1}, k_{2} f_{1} k_{2}, \quad f_{1} k_{2} f_{1}$, & \\
& $k_{2} f_{1} f_{1}, k_{2} f_{1} f_{2}, \quad f_{1} i_{1} f_{*}, f_{1} i_{2} f_{*}, f_{2} i_{2} f_{*}$ & 10 \\
\hline 4 & $f_{1} i_{2} k_{*} i_{*}, \quad f_{1} k_{2} i_{*} k_{*}, \quad k_{2} f_{1} k_{1} i_{*}, k_{2} f_{1} k_{2} i_{*}$, & 3 \\
\hline & $k_{2} f_{1} i_{1} k_{*}, k_{2} f_{1} i_{2} k_{*}, \quad f_{1} k_{2} i_{*} f_{*}$, & \\
\hline
\end{tabular}

It is also straightforward to check, on a case-by-case basis, that the 60 operators in $\mathcal{K F}_{2}^{0}$ are distinct, in the sense that for any $\omega_{1}, \omega_{2}$ as in the table above with $\omega_{1} \neq \omega_{2}$, there exists a subset $A^{\omega_{1}, \omega_{2}}$ of some 2 -topological space $\left(X, \tau_{1}, \tau_{2}\right)$ for which $\omega_{1} A^{\omega_{1}, \omega_{2}} \neq \omega_{2} A^{\omega_{1}, \omega_{2}}$. 
Combining this observation with the simple lemma below, we obtain the stronger fact that there exists a 2-topological space with an initial subset $A$ which distinguishes all of the operators in $\mathcal{K F}_{2}$ simultaneously.

Lemma 3.2. Suppose that for any distinct pair of operators $\omega_{1}, \omega_{2} \in \mathcal{K} \mathcal{F}_{n}^{0}$, there exists a saturated $n$-topological space $X^{\omega_{1}, \omega_{2}}$ and a subset $A^{\omega_{1}, \omega_{2}} \subseteq X^{\omega_{1}, \omega_{2}}$ in which $\omega_{1} A^{\omega_{1}, \omega_{2}} \neq \omega_{2} A^{\omega_{1}, \omega_{2}}$. Then there exist a saturated $n$-topological space $X$ and a subset $A \subseteq X$ such that $\omega_{1} A \neq \omega_{2} A$, for each pair of distinct operators $\omega_{1}, \omega_{2} \in$ $\mathcal{K} \mathcal{F}_{n}^{0}$.

Proof. If the assumption is true, then we can construct the $n$-topological disjoint union $X=\bigcup_{\substack{\omega_{1}, \omega_{2} \in \mathcal{K F}_{n}^{0} \\ \omega_{1} \neq \omega_{2}}} X^{\omega_{1}, \omega_{2}}$ and form the initial set $A=\bigcup_{\omega_{1} \neq \omega_{2}} A^{\omega_{1}, \omega_{2}}$. Then for any operators $\omega_{1} \neq \omega_{2}$ in $\mathcal{K} \mathcal{F}_{n}^{0}$, we have $\left(\omega_{1} A\right) \Delta\left(\omega_{2} A\right) \supseteq\left(\omega_{1} A^{\omega_{1}, \omega_{2}}\right) \Delta\left(\omega_{2} A^{\omega_{1}, \omega_{2}}\right) \neq$ $\emptyset$ (where $\Delta$ denotes the symmetric difference), and therefore $\omega_{1} A \neq \omega_{2} A$.

Despite the preceding, we would like to follow the tradition of the closurecomplement theorem by exhibiting an explicit initial set $A \subseteq \mathbb{R}$ which simultaneously distinguishes the operators in $\mathcal{K F}_{2}$.

Example 3.3 (An Initial Set For $\mathcal{K F}_{2}$ in the Usual/Sorgenfrey Line). We consider the 2-topological space $\left(\mathbb{R}, \tau_{1}, \tau_{2}\right)$ where $\tau_{1}=\tau_{s}$ is the Sorgenfrey topology and $\tau_{2}=\tau_{u}$ is the usual Euclidean topology. We define

$$
\begin{aligned}
& S^{0}=\bigcup_{k=0}^{\infty}\left(\frac{1}{3^{2 k+1}}, \frac{1}{3^{2 k}}\right), S^{1}=\bigcup_{k=0}^{\infty}\left[\frac{1}{3^{2 k+1}}, \frac{1}{3^{2 k}}\right), \\
& S^{2}=\bigcup_{k=0}^{\infty}\left[\frac{1}{3^{2 k+1}}, \frac{1}{3^{2 k}}\right], S^{*}=\bigcup_{k=0}^{\infty}\left(\frac{1}{3^{2 k+1}}, \frac{1}{3^{2 k}}\right], \\
& T^{0}=\bigcup_{k=1}^{\infty}\left(\frac{1}{3^{2 k}}, \frac{2}{3^{2 k}}\right), T^{1}=\bigcup_{k=1}^{\infty}\left[\frac{1}{3^{2 k}}, \frac{2}{3^{2 k}}\right), \\
& T^{2}=\bigcup_{k=1}^{\infty}\left[\frac{1}{3^{2 k}}, \frac{2}{3^{2 k}}\right], T^{*}=\bigcup_{k=1}^{\infty}\left(\frac{1}{3^{2 k}}, \frac{2}{3^{2 k}}\right],
\end{aligned}
$$

and we take the following initial set:

$$
\begin{gathered}
A=\left(S^{0} \cap \mathbb{Q}\right) \cup T^{1} \cup\left(\left(2-S^{0}\right) \cap \mathbb{Q}\right) \cup\left(2-T^{0}\right) \cup((2,3) \cap \mathbb{Q}) \cup\{4\} \cup(5,6) \cup(6,7) \\
\cup \bigcup_{n=0}^{\infty}\left(\left(\frac{1}{2^{n+2}} S^{2}+8-\frac{1}{2^{n}}\right) \cap \mathbb{Q}\right) \cup \bigcup_{n=0}^{\infty}\left(\frac{1}{2^{n+2}} S^{2}+10-\frac{1}{2^{n}}\right) \cup(10,11) .
\end{gathered}
$$

It is possible to verify by hand that applying the 60 operators of the monoid $\mathcal{K F}_{2}^{0}$ to $A$ yields 60 distinct sets. The results of such a computation appear in a previous draft of this paper (posted August 3, 2019) accessible via arXiV.org. Bowron, in private communication, has also provided us with an elegant and brief computer-assisted verification. Rather than presenting such a verification here, we 
will turn to a stronger result, by first considering the natural partial order on the monoid $\mathcal{K F}_{n}^{0}$.

The partial order is defined as follows: for every $o_{1}, o_{2} \in \mathcal{K} \mathcal{F}_{n}^{0}$,

$$
o_{1} \leq o_{2} \quad \text { if and only if } \quad o_{1} A \subseteq o_{2} A \text { for every } A \subseteq X .
$$

The partial orderings on $\mathcal{K}_{1}^{0}, \mathcal{K F}_{1}^{0}$ (see Figure 1), and other related monoids have been diagrammed by various authors; see especially [6] and [4. It is clear that $\mathcal{K F}_{n}$ has a minimal element 0 and a maximal element $k_{n}$, and that $0 \leq i_{n} \leq$ $\ldots \leq i_{1} \leq \mathrm{Id} \leq k_{1} \leq \ldots \leq k_{n}$. It is also clear that for any set operator $o$ we have $i_{j} o \leq o \leq k_{j} o$.

By the definition, for any operators $o_{1}, o_{2}, o_{3}$, if $o_{1} \leq o_{2}$ then $o_{1} o_{3} \leq o_{2} O_{3}$, so order is preserved by multiplication on the right. The operators $i_{j}$ and $k_{j}$ $(1 \leq j \leq n)$ are also left order-preserving in the sense that if $o_{1} \leq o_{2}$, then $i_{j} O_{1} \leq i_{j} o_{2}$ and $k_{j} o_{1} \leq k_{j} o_{2}$. On the other hand, $f_{j}$ is not left order-preserving in general.

Example 3.4 (Exhibiting the Partial Order on $\mathcal{K F}_{2}^{0}$ ). We will now show there exists a set $A$ in a 2-topological space with the property that $o_{1} \leq o_{2}$ if and only if $o_{1} A \subseteq o_{2} A$, for each $o_{1}, o_{2} \in \mathcal{K F}_{2}^{0}$. In particular, the 60 operators of $\mathcal{K F}_{2}^{0}$ applied to $A$ yield 60 distinct sets.



FiguRE 1. The partial ordering on the 17 operators of $\mathcal{K} \mathcal{F}_{1}^{0}$, which was computed by Gaida and Eremenko but did not appear in the printed version of their article [5; see also [4. Subscripts are omitted from the notation since only one topology is involved. 
We first present a list of apparently non-obvious inequalities in the partially ordered set $\mathcal{K F}_{2}^{0}$.

Proposition 3.5. The following relations hold in any saturated 2-topological space $\left(X, \tau_{1}, \tau_{2}\right)$ :

(a) $f_{1} i_{1} \leq f_{1} i_{2}$ and $f_{1} k_{1} \leq f_{1} k_{2}$

(b) $f_{1} k_{1} i_{*} \leq f_{1} i_{2} k_{*} i_{*}$ and $f_{1} i_{1} k_{*} \leq f_{1} k_{2} i_{*} k_{*}$;

(c) $f_{1} f_{1} \leq f_{1} k_{2} f_{1}$;

(d) $f_{1} k_{2} f_{1} \leq f_{1} f_{2}$;

(e) $f_{1} k_{2} i_{*} k_{*} \leq f_{1} k_{2}$ and $f_{1} i_{2} k_{*} i_{*} \leq f_{1} i_{2}$;

(f) $f_{1} k_{2} \leq f_{1} f_{2}$.

Proof. For (a), we have $f_{1} i_{1}=k_{1} i_{*} \wedge c i_{1}=k_{1} i_{*} \wedge k_{1} c \leq k_{1} i_{*} \wedge k_{2} c=k_{1} i_{*} \wedge c i_{2}=$ $f_{1} i_{2}$, and the second statement follows in a dual way, because we can multiply the first inequality on the right by $c$, and get $f_{1} k_{1}=f_{1} i_{1} c \leq f_{1} i_{2} c=f_{1} k_{2}$.

For (b), we have

$$
\begin{aligned}
f_{1} k_{1} i_{*} & =k_{1} k_{1} i_{*} \wedge k_{1} c k_{1} i_{*}=k_{1} i_{*} \wedge k_{1} i_{*} k_{*} c . \\
f_{1} i_{2} k_{*} i_{*} & =k_{1} i_{*} k_{*} i_{*} \wedge k_{1} c i_{2} k_{*} i_{*}=k_{1} i_{*} \wedge k_{2} i_{*} k_{*} c .
\end{aligned}
$$

The second statement follows dually.

For (c), we compute $f_{1} f_{1}=f_{1} \wedge c i_{1} f_{1}$ and $f_{1} k_{2} f_{1}=k_{2} f_{1} \wedge c i_{1} k_{*} f_{1}=k_{2} f_{1} \wedge c i_{1} f_{1}$, so the inequality $f_{1} f_{1} \leq f_{1} k_{2} f_{1}$ follows from $f_{1} \leq k_{2} f_{1}$.

For $(\mathrm{d})$, compute $f_{1} f_{1}=f_{1} \wedge c i_{1} f_{1}$ and $f_{1} k_{2} f_{1}=k_{2} f_{1} \wedge c i_{1} k_{*} f_{1}=k_{2} f_{1} \wedge c i_{1} f_{1}$, so the inequality $f_{1} f_{1} \leq f_{1} k_{2} f_{1}$ follows from $f_{1} \leq k_{2} f_{1}$.

For (e), compute $f_{1} k_{2} i_{*} k_{*}=k_{1} k_{2} i_{*} k_{*} \wedge k_{1} c k_{2} i_{*} k_{*}=k_{2} i_{*} k_{*} \wedge k_{1} i_{*} c \leq k_{2} \wedge k_{1} c k_{2}=$ $f_{1} k_{2}$.

Lastly, for (f), note that $k_{1} i_{*} \leq k_{1} \leq k_{2}$. Hence $f_{1} k_{2}=k_{1} k_{2} \wedge k_{1} c k_{2}=k_{2} \wedge k_{1} i_{*} c=$ $k_{2} \wedge\left(k_{2} c \wedge k_{1} i_{*} c\right) \leq\left[\left(k_{2} \wedge k_{2} c\right) \wedge k_{1} i_{*} c\right] \vee\left[\left(k_{2} \wedge k_{2} c\right) \wedge k_{1} i_{*}\right]=\left(k_{2} \wedge k_{2} c\right) \wedge\left(k_{1} i_{*} c \vee\right.$ $\left.k_{1} i_{*}\right)=f_{2} \wedge\left(k_{1} c k_{*} \vee k_{1} c k_{*} c\right)=f_{2} \wedge k_{1}\left(c k_{2} \vee c k_{2} c\right)=k_{1} f_{2} \wedge k_{1} c\left(k_{2} \wedge k_{2} c\right)=f_{1} f_{2}$.

Using the inequalities in the proposition, together with the facts that closure and interior are left order-preserving, and all operators are right order-preserving, we obtain the diagram of the partially ordered set $\mathcal{K} \mathcal{F}_{2}^{0}$ depicted in Figure 2.

To show that no further inequalities hold in general, we define a partition $P=$ $\left\{P_{0}, \ldots, P_{12}\right\}$ of $\mathbb{R}^{+}$such that for each inequality $o_{1} \leq o_{2}\left(o_{1}, o_{2} \in \mathcal{K F}_{2}^{0}\right)$ not implied by Figure 2, there exist integers $0 \leq \alpha_{1}<\cdots<\alpha_{n} \leq 12(1 \leq n \leq 12)$ satisfying $o_{1}\left(P_{\alpha_{1}} \cup \cdots \cup P_{\alpha_{n}}\right) \nsubseteq o_{2}\left(P_{\alpha_{1}} \cup \cdots \cup P_{\alpha_{n}}\right)$ in $\left(\mathbb{R}^{+}, \bar{\tau}_{1}, \tau_{2}\right)$ where $\tau_{1}=\tau_{s}$ is the Sorgenfrey topology and $\tau_{2}=\tau_{u}$ is the usual Euclidean topology.

The partition $\left\{\pi_{1}, \ldots, \pi_{8}\right\}$ of $(0,1]$ is defined as follows:

$$
\begin{array}{lll}
\pi_{1}=\bigcup_{n=1}^{\infty}\left\{\frac{1}{3^{2 n}}\right\} & \pi_{3}=\bigcup_{n=1}^{\infty}\left\{\frac{2}{3^{2 n}}\right\} & \pi_{5}=\bigcup_{n=1}^{\infty}\left\{\frac{1}{3^{2 n-1}}\right\} \\
\pi_{2}=\bigcup_{n=1}^{\infty}\left(\frac{1}{3^{2 n}}, \frac{2}{3^{2 n}}\right) & \pi_{4}=\bigcup_{n=1}^{\infty}\left(\frac{2}{3^{2 n}}, \frac{1}{3^{2 n-1}}\right) & \pi_{6}=\bigcup_{n=1}^{\infty}\left(\frac{1}{3^{2 n-1}}, \frac{1}{3^{2 n-2}}\right) \cap \mathbb{Q} \\
& \pi_{7}=\bigcup_{n=1}^{\infty}\left(\frac{1}{3^{2 n-1}}, \frac{1}{3^{2 n-2}}\right) \backslash \mathbb{Q} \\
\pi_{8}=\{1\} .
\end{array}
$$




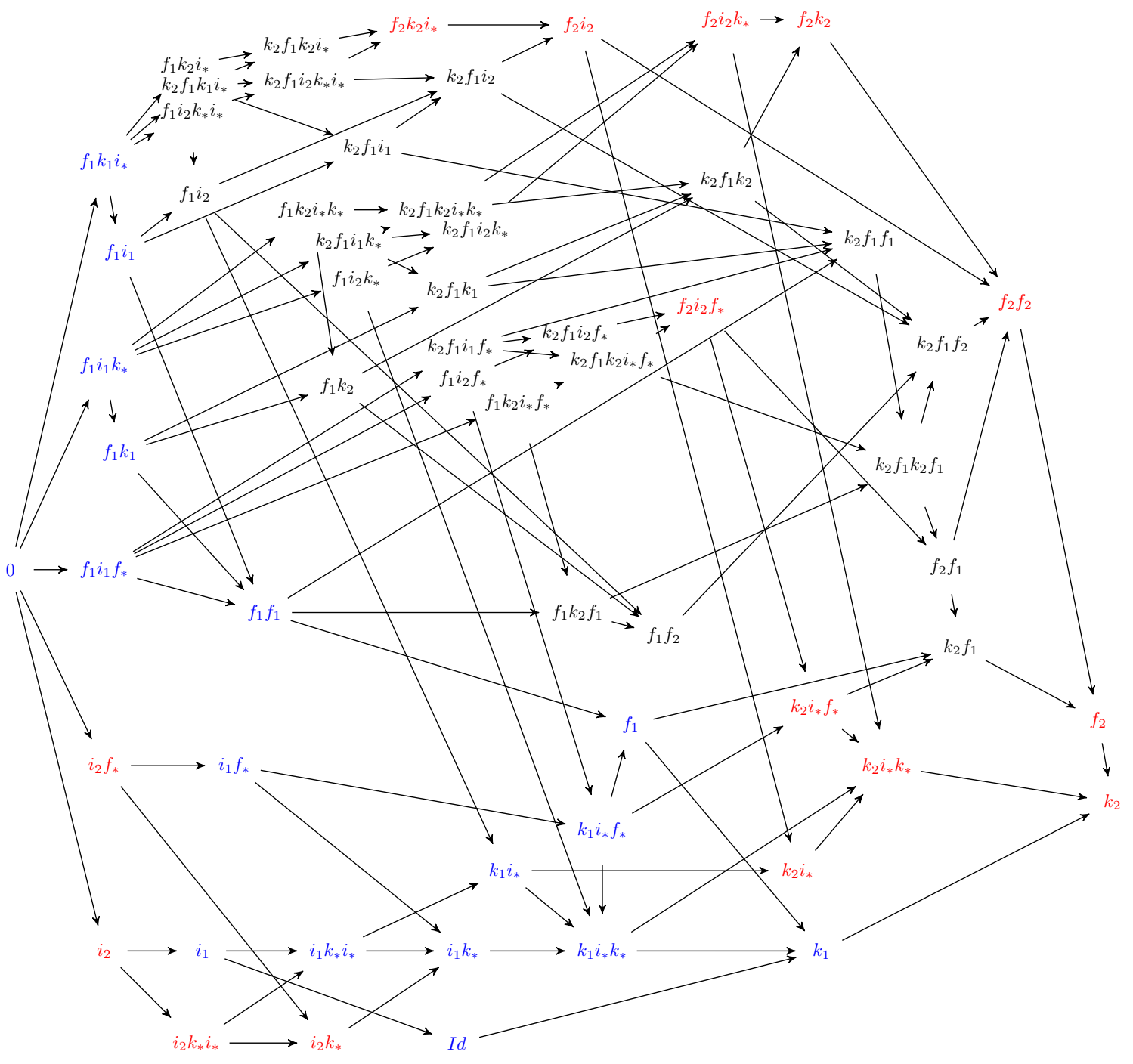

Figure 2. The partial ordering on $\mathcal{K F}_{2}^{0}$. The blue operators are operators that can be built using exclusively the $\tau_{1}$ topology. The red operators are operators built using the topology $\tau_{2}$ that cannot also be built using $\tau_{1}$. The black operators are those built using a combination of both topologies. 
For $1 \leq j \leq 8$, let $P_{j}=\bigcup_{n=0}^{\infty}\left(1-\frac{1}{2^{n}}+\frac{1}{2^{n+2}} \pi_{j}\right)$. Thus

$$
P_{1} \cup \cdots \cup P_{8}=\left(0, \frac{1}{4}\right] \cup\left(\frac{1}{2}, \frac{5}{8}\right] \cup\left(\frac{3}{4}, \frac{13}{16}\right] \cup \cdots .
$$

To complete the definition of $P$, set

$$
\begin{array}{ll}
P_{0}=\left\{1-\frac{1}{2^{n}}: n=1,2, \ldots\right\} & P_{10}=(1, \infty) \cap \mathbb{Q} \\
P_{9}=\left(\frac{1}{4}, \frac{1}{2}\right) \cup\left(\frac{5}{8}, \frac{3}{4}\right) \cup\left(\frac{13}{16}, \frac{7}{8}\right) \cup \ldots &
\end{array}
$$

Then each of the following equations holds in $\left(\mathbb{R}^{+}, \tau_{1}, \tau_{2}\right)$ :

$$
\begin{aligned}
k_{1} P_{0} & =P_{0} & k_{2} P_{0} & =P_{0} \cup P_{10} \\
k_{1} P_{1} & =P_{0} \cup P_{1} & k_{2} P_{1} & =P_{0} \cup P_{1} \cup P_{10} \\
k_{1} P_{2} & =P_{0} \cup P_{1} \cup P_{2} & k_{2} P_{2} & =P_{0} \cup P_{1} \cup P_{2} \cup P_{3} \cup P_{10} \\
k_{1} P_{3} & =P_{0} \cup P_{3} & k_{2} P_{3} & =P_{0} \cup P_{3} \cup P_{10} \\
k_{1} P_{4} & =P_{0} \cup P_{3} \cup P_{4} & k_{2} P_{4} & =P_{0} \cup P_{3} \cup P_{4} \cup P_{5} \cup P_{10} \\
k_{1} P_{5} & =P_{0} \cup P_{5} & k_{2} P_{5} & =P_{0} \cup P_{5} \cup P_{10} \\
k_{1} P_{6} & =P_{0} \cup P_{5} \cup P_{6} \cup P_{7} & k_{2} P_{6} & =P_{0} \cup P_{1} \cup P_{5} \cup P_{6} \cup P_{7} \cup P_{8} \cup P_{10} \\
k_{1} P_{7} & =P_{0} \cup P_{5} \cup P_{6} \cup P_{7} & k_{2} P_{7} & =P_{0} \cup P_{1} \cup P_{5} \cup P_{6} \cup P_{7} \cup P_{8} \cup P_{10} \\
k_{1} P_{8} & =P_{8} & k_{2} P_{8} & =P_{8} \cup P_{10} \\
k_{1} P_{9} & =P_{8} \cup P_{9} & k_{2} P_{9} & =P_{0} \cup P_{8} \cup P_{9} \cup P_{10} \\
k_{1} P_{10} & =P_{10} & k_{2} P_{10} & =P_{10} \\
k_{1} P_{11} & =P_{10} \cup P_{11} \cup P_{12} & k_{2} P_{11} & =P_{10} \cup P_{11} \cup P_{12} \\
k_{1} P_{12} & =P_{10} \cup P_{11} \cup P_{12} & k_{2} P_{12} & =P_{10} \cup P_{11} \cup P_{12} .
\end{aligned}
$$

Using these equations, all inclusions not implied by Figure 2 may be eliminated computationally. Bowron has written the following $\mathrm{C}$ program and Python script which verify the eliminations:

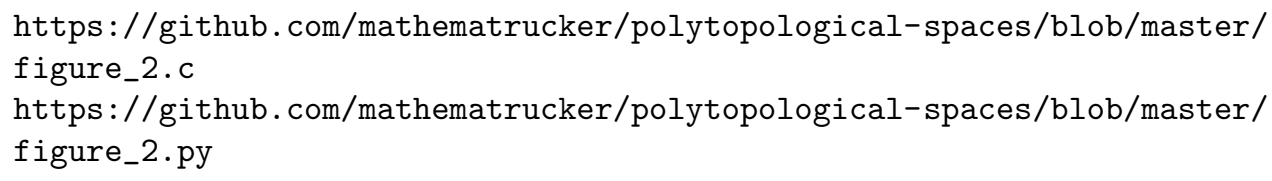

Following Lemma 3.2, we may take the disjoint union of all possible sets of the form $P_{\alpha_{1}} \cup \cdots \cup P_{\alpha_{n}}$ to obtain an initial set $A$ with the property that if $o_{1}, o_{2} \in \mathcal{K} \mathcal{F}_{2}^{0}$ and $o_{1} \not \leq o_{2}$, then $o_{1} A \nsubseteq o_{2} A$. Consequently, $o_{1} \leq o_{2}$ if and only if $o_{1} A \subseteq o_{2} A$, for all $o_{1}, o_{2} \in \mathcal{K F}_{2}^{0}$.

\section{The General Case}

We are ready to solve the closure-complement-frontier problem in the general setting of a saturated $n$-topological space where $n$ is arbitrary. The surprising fact which underlies our computation is that every reduced word in $\mathcal{K F}_{n}^{0}$ has length $\leq 5$, and in fact has the same form as one of the reduced words which we already computed in Section 3 for $\mathcal{K F}_{2}^{0}$.

In order to prove this observation we define the following subsets of $\mathcal{K F}_{n}^{0}$ : 


$$
\begin{aligned}
K & =\left\{k_{j}: 1 \leq j \leq n\right\} \\
I & =\left\{i_{j}: 1 \leq j \leq n\right\} \\
F & =\left\{f_{j}: 1 \leq j \leq n\right\}
\end{aligned}
$$

We also allow the formation of product sets in $\mathcal{K F}_{n}^{0}$ in the usual way, so we may write, for example, $K F I=\{k f i: k \in K, i \in I, f \in F\}$. So if $n=2$, we could explicitly write

$$
K F I=\left\{f_{1} i_{1}, f_{1} i_{2}, k_{2} f_{1} i_{1}, k_{2} f_{1} i_{2}, f_{2} i_{*}\right\}
$$

We will now adopt a notational convention which will not lead to ambiguity in the context of this paper, and which will help us clearly delineate word types in $\mathcal{K} \mathcal{F}_{n}^{0}$. Suppose $E$ is a set which is the $n$-times product of the sets $K, I$, and $F$ (in any order). Then we denote by $(E)_{r}$ the set of all reduced words $\omega \in E$, i.e. those which do not admit any representation as a word of length $<n$. So, under this convention, if $n=2$ we would have

$$
(K F I)_{r}=\left\{k_{2} f_{1} i_{1}, k_{2} f_{1} i_{2}\right\} .
$$

We are now ready to prove our main Theorem 1.1, which is a consequence of the more detailed theorem below.

Theorem 4.1. Let $\left(X, \tau_{1}, \ldots, \tau_{n}\right)$ be a saturated $n$-topological space. Then $\mathcal{K F}_{n}^{0}$ is contained in the union of the sets in the left-hand column of the table below. The number of distinct elements in each such set is at most as listed in the right-hand column.

\begin{tabular}{|c|r|}
\hline Word-Type & Number of Words \\
\hline$\{\mathrm{Id}\}$ & 1 \\
IF $=\{0\}$ & 1 \\
$I$ & $n$ \\
$K$ & $n$ \\
$I K$ & $n$ \\
$K I$ & $n$ \\
$I K I$ & $n$ \\
$K I K$ & $n$ \\
$F$ & $n$ \\
$I F$ & $n$ \\
$F F$ & $n^{2}$ \\
$F I$ & $n+\left(\begin{array}{c}n \\
2\end{array}\right)$ \\
$F K$ & $n+\left(\begin{array}{c}n \\
2\end{array}\right)$ \\
$F I F$ & $n+\left(\begin{array}{c}n \\
2\end{array}\right)$ \\
$K I F$ & $n$ \\
$F I K$ & $n+\left(\begin{array}{c}n \\
2\end{array}\right)$ \\
\hline
\end{tabular}

\begin{tabular}{|c|r|}
\hline Word-Type & Number of Words \\
\hline$F K I$ & $n+\left(\begin{array}{c}n \\
2\end{array}\right)$ \\
$(K F)_{r}$ & $\left(\begin{array}{l}n \\
2\end{array}\right)$ \\
$(K F K)_{r}$ & $2\left(\begin{array}{c}n+1 \\
3\end{array}\right)$ \\
$(K F I)_{r}$ & $2\left(\begin{array}{c}n+1 \\
3\end{array}\right)$ \\
$(K F F)_{r}$ & $\left(\begin{array}{c}n \\
2\end{array}\right) \cdot n$ \\
$(F K F)_{r}$ & $\left(\begin{array}{l}n \\
2\end{array}\right)+2\left(\begin{array}{l}n \\
3\end{array}\right)$ \\
$(F I K I)_{r}$ & $\left(\begin{array}{l}n \\
2\end{array}\right)$ \\
$(F K I K)_{r}$ & $\left(\begin{array}{l}n \\
2\end{array}\right)$ \\
$(F K I F)_{r}$ & $\left(\begin{array}{l}n \\
2\end{array}\right)$ \\
$(K F I K)_{r}$ & $2\left(\begin{array}{c}n+1 \\
3\end{array}\right)$ \\
$(K F K I)_{r}$ & $2\left(\begin{array}{c}n+1 \\
3\end{array}\right)$ \\
$(K F I F)_{r}$ & $2\left(\begin{array}{c}n+1 \\
3\end{array}\right)$ \\
$(K F K F)_{r}$ & $\left(\begin{array}{c}n \\
2\end{array}\right)+5\left(\begin{array}{c}n \\
3\end{array}\right)+5\left(\begin{array}{c}n \\
4\end{array}\right)$ \\
$(K F I K I)_{r}$ & $\left(\begin{array}{c}n \\
2\end{array}\right)+2\left(\begin{array}{c}n \\
3\end{array}\right)$ \\
$(K F K I K)_{r}$ & $\left(\begin{array}{c}n \\
2\end{array}\right)+2\left(\begin{array}{c}n \\
3\end{array}\right)$ \\
$(K F K I F)_{r}$ & $\left(\begin{array}{l}n \\
2\end{array}\right)+2\left(\begin{array}{c}n \\
3\end{array}\right)$ \\
\hline
\end{tabular}


Consequently, the number of elements of $\mathcal{K F}_{n}^{0}$ is at most

$$
\begin{aligned}
p(n) & =5\left(\begin{array}{l}
n \\
4
\end{array}\right)+10\left(\begin{array}{c}
n+1 \\
3
\end{array}\right)+13\left(\begin{array}{l}
n \\
3
\end{array}\right)+(n+14)\left(\begin{array}{l}
n \\
2
\end{array}\right)+n^{2}+14 n+2 \\
& =\frac{5}{24} n^{4}+\frac{37}{12} n^{3}+\frac{79}{24} n^{2}+\frac{101}{12} n+2
\end{aligned}
$$

and the number of elements of $\mathcal{K F}_{n}$ is at most $2 p(n)$.

Proof. Let $\mathcal{X}$ be the union of all of the sets in the table above, so we want to prove $\mathcal{K F}_{n}^{0} \subseteq \mathcal{X}$. For this, it suffices to check that (A) for each set $E$ listed in the table above, and for each $1 \leq x \leq n$, we have $k_{x} E, i_{x} E, f_{x} E \subseteq \mathcal{X}$. Our second goal (B) is to establish the listed upper bound for the cardinality of each set.

We can begin the verification by making these observations:

- Every 0- and 1-letter word type in $\mathcal{K F}_{n}^{0}$ (i.e. the elements of $\{\operatorname{Id}\}, K, I$, and $F$ ) is accounted for in the table.

- There are $3^{2}=9$ possible 2-letter word types. By Lemma 2.3 we have $I I=I$ and $K K=K$, and the other seven possible types are accounted for on the table. So all elements of $\mathcal{K F}_{n}^{0}$ which admit a word representation of length $\leq 2$ are contained in $\mathcal{X}$.

- There are $3^{3}=27$ possible 3 -letter word types. Ten of these reduce to 2-letter words using $I I=I$ and $K K=K$, which by the previous bullet point, are already accounted for in the table. At most seventeen types remain, and among these, we know that $I F K=I F I=0$ by Lemma 2.10, while $F F K=K F K$, $F F I=K F I$, and $F F F=K F F$ by Lemma 2.11. Also $I K F=I F$ by Lemmas 2.4 and 2.3 , and since $F \subseteq K F$, we have $I F F \subseteq I F K F \subseteq\{0\} F \subseteq\{0\}$. This leaves eleven other possible 3-letter word types, each of which is listed in the table. Therefore, all elements of $\mathcal{K F}_{n}^{0}$ which admit a word representation of length $\leq 3$ are already contained in a subset listed in the table.

By the last bullet point above, we see that whenever $E$ consists of $\leq 2$-letter words, then indeed we have $k_{x} E, i_{x} E, f_{x} E \subseteq \mathcal{X}$ for each $1 \leq x \leq n$, which establishes (A) for the sets $\{\operatorname{Id}\}, K, I, I K, K I, F, I F F F, F I, F K$, and $(K F)_{r}$. (A) is also immediate for the set $\{0\}$.

The cardinality bounds (B) are immediate for the sets $\{\mathrm{Id}\},\{0\}, K, I, I K, K I$, $F$, and FF. By Lemma 2.5 the set $I F$ consists of words of the form $i_{x} f_{*}(1 \leq x \leq$ $n$ ), of which there are $n$ many. The set $(K F)_{r}$ consists of elements of the form $k_{x} f_{y}$ which do not reduce to 1-letter representations; by Lemma 2.3 it is necessary that $x>y$. There are $\left(\begin{array}{l}n \\ 2\end{array}\right)$ many pairs $(x, y)$ with $x>y$, so $\#(K F)_{r} \leq\left(\begin{array}{l}n \\ 2\end{array}\right)$. Lastly, by Lemma 2.6, the set $F K$ consists of words of the form $f_{x} k_{y}$ where $1 \leq x \leq y \leq n$; there are $n+\left(\begin{array}{l}n \\ 2\end{array}\right)$ many such pairs $(x, y)$, and thus $F K$ consists of no more than $n+\left(\begin{array}{l}n \\ 2\end{array}\right)$ elements. A similar argument yields the same number for $F I$.

So to finish the proof, it remains only to check (A) and (B) for those sets $E$ which consist of words of length $\geq 3$.

The sets $I K I$ and $K I K$. By Lemma 2.4, every element of $I K I$ has the form $i_{y} k_{*} i_{*}$ for some $1 \leq y \leq n$, and thus $\# I K I \leq n$, establishing (B). Note that for any 
$1 \leq x \leq n$, we have $k_{x} i_{y} k_{*} i_{*}=k_{x} i_{*} k_{*} i_{*}=k_{x} i_{*}$ by Lemma 2.3 , so $k_{x} I K I \subseteq K I \subseteq$ $\mathcal{X}$. Also $i_{x} i_{y} k_{*} i_{*}=i_{\max (x, y)} k_{*} i_{*}$ by Lemma 2.3 , so $i_{x} I K I \subseteq I K I \subseteq \mathcal{X}$. The word $f_{x} i_{y} k_{*} i_{*}$ either reduces to a $\leq 3$-letter word, in which case it is a member of $\mathcal{X}$ by our previous remarks; or it does not reduce, in which case $f_{x} i_{y} k_{*} i_{*} \in(F I K I)_{r} \subseteq \mathcal{X}$. This establishes (A), and the arguments are similar for $K I K$.

The set FIF. By Lemma 2.5 every element of FIF has the form $f_{y} i_{z} f_{*}$, so $F I F \subseteq F I f_{1}$. Therefore (B) \#FIF $\leq \# F I \leq n+\left(\begin{array}{l}n \\ 2\end{array}\right)$. For $1 \leq x \leq n$, we have either $k_{x} f_{y} i_{z} f_{*} \in(K F I F)_{r}$ or $k_{x} f_{y} i_{z} f_{*}$ reduces to a shorter word; in either case we obtain $k_{x} f_{y} i_{z} f_{*} \in \mathcal{X}$ and hence $k_{x} F I F \subseteq \mathcal{X}$. By Lemma 2.10 we see $i_{x} f_{y} i_{z} f_{*}=0 f_{*}=0 \in \mathcal{X}$, and by Lemma 2.11 we see $f_{x} f_{y} i_{z} f_{*}=k_{x} f_{y} i_{z} f_{*} \in \mathcal{X}$, establishing (A).

The set KIF. By Lemmas 2.4 and 2.5 every element of $K I F$ has the form $k_{y} i_{*} f_{*}$, where $1 \leq y \leq n$, so (B) holds. For any $1 \leq x \leq n, k_{x} k_{y} i_{*} f_{*}=$ $k_{\max (x, y)} i_{*} f_{*} \in K I F \subseteq \mathcal{X}$, and $i_{x} k_{y} i_{*} f_{*}=i_{x} k_{*} i_{*} k_{*} f_{*}=i_{x} k_{*} f_{*} \in I K F \subseteq \mathcal{X}$. The word $f_{x} k_{y} i_{*} f_{*}$ either reduces to a $\leq 3$-letter word or else lies in $(F K I F)_{r}$; in either case it lies in $\mathcal{X}$, establishing (A).

The sets $F I K$ and $F K I$. Elements of $F I K$ have the form $f_{y} i_{z} k_{*}$, so $F I K=$ $F I k_{1}$, and (B) $\# F I K \leq \# F I \leq n+\left(\begin{array}{l}n \\ 2\end{array}\right)$. For (A), note that for any $x$, the word $k_{x} f_{y} i_{z} k_{*}=f_{x} f_{y} i_{z} k_{*}$ either reduces to a $\leq 3$ letter word or else lies in $(K F I K)_{r}$, so it lies in $\mathcal{X}$, while $i_{x} F I K=\{0\} K=\{0\} \subseteq \mathcal{X}$ as well. The arguments are similar for $F K I$.

The sets $(K F K)_{r}$ and $(K F I)_{r}$. Elements of $(K F K)_{r}$ have the form $k_{y} f_{z} k_{w}$. To establish (A), we note that for $1 \leq x \leq n$, we have $k_{x} k_{y} f_{z} k_{w}=k_{\max (x, y)} f_{z} k_{w}$ by Lemma 2.3. $i_{x} k_{y} f_{z} k_{w}=i_{x} k_{*} f_{z} k_{w}=i_{x} f_{z} k_{w}$ by Lemma 2.4. and $f_{x} k_{y} f_{z} k_{w}=$ $k_{\max (x, y)} f_{z} k_{w}$ by Lemma 2.12 Then all three words admit representations of length $\leq 3$, and therefore lie in $\mathcal{X}$.

For $(\mathbf{B})$, since $k_{y} f_{z} k_{w}$ cannot be written with $\leq 2$ letters, by Lemma 2.3 it is necessary that $y>z$. Also, by Lemma 2.6, we may assume that $w \geq z$. The number of triples $(y, z, w)$ with $y>z$ and $z \leq w$ may be found by the following reasoning: either $z=w$ or $z \neq w$. If $z=w$, we find $\left(\begin{array}{l}n \\ 2\end{array}\right)$ many triples $(y, z, z)$ with $y>z$. If $z \neq w$, either $w=y$ or $w \neq y$. If $w=y$ we again obtain $\left(\begin{array}{l}n \\ 2\end{array}\right)$ many triples $(y, z, y)$. If $w \neq y$, then there are $\left(\begin{array}{l}n \\ 3\end{array}\right)$ many sets of distinct numbers $\{y, z, w\}$ where $z$ is minimal; these each yield two choices of ordered triples $(y, z, w)$ or $(w, z, y)$. So the cardinality of $(K F K)_{r}$ is no more than $\left(\begin{array}{l}n \\ 2\end{array}\right)+\left(\begin{array}{l}n \\ 2\end{array}\right)+2\left(\begin{array}{c}n \\ 3\end{array}\right)=2\left(\begin{array}{c}n+1 \\ 3\end{array}\right)$. The arguments are similar for $(K F I)_{r}$.

The set $(K F F)_{r}$. Elements of $(K F F)_{r}$ have the form $k_{y} f_{z} f_{w}$, which can be rewritten as $k_{y} f_{z} k_{w} f_{w}$; thus the arguments to establish (A) are exactly analogous to those given for the case of $(K F K)_{r}$. For (B), we note that since $k_{y} f_{z} f_{w}$ cannot be written as a word of length $\leq 2$, it must be the case that $k_{y} f_{z} \in(K F)_{r}$. Therefore $\#(K F F)_{r} \leq \#(K F)_{r} \cdot \# F=\left(\begin{array}{l}n \\ 2\end{array}\right) \cdot n$. 
The set $(F K F)_{r}$. Elements of $(F K F)_{r}$ have the form $f_{y} k_{z} f_{w}$. To establish (A), note that for $1 \leq x \leq n$, we have $k_{x} f_{y} k_{z} f_{w}=f_{x} f_{y} k_{z} f_{w}$ by Lemma 2.11 and this word either admits a word representation of length $\leq 3$ and therefore lies in $\mathcal{X}$, or else it lies in $(K F K F)_{r} \subseteq \mathcal{X}$. Also $i_{x} f_{y} k_{z} f_{w}=0 f_{w}=0 \in \mathcal{X}$.

For (B), since $f_{y} k_{z} f_{w}$ cannot be written with $\leq 2$ letters, by Lemma 2.8 it is necessary that $z>y$ and $z>w$. We have either $y=w$ or $y \neq w$. If $y=w$ we are looking for triples of the form $(y, z, y)$ with $z>y$, of which there $\left(\begin{array}{l}n \\ 2\end{array}\right)$ many. If $y \neq w$, we find $\left(\begin{array}{l}n \\ 3\end{array}\right)$ many sets $\{y, z, w\}$ of distinct numbers where $z$ is maximal; each of these yields two choices of ordered triples $(y, z, w)$ or $(w, z, y)$. So the cardinality of $(F K F)_{r}$ is no more than $\left(\begin{array}{l}n \\ 2\end{array}\right)+2\left(\begin{array}{l}n \\ 3\end{array}\right)$.

At this point, we pause to observe the following: combining all the arguments in the previous parts, we have shown that if $o \in \mathcal{K F}_{n}^{0}$ admits any representation as a word of length $\leq 3$, then for every $1 \leq x \leq n$, we have $k_{x} o, i_{x} o, f_{x} o \in \mathcal{X}$. All words of length $\leq 4$ have this form, so put in other words, we have now shown:

- All elements of $\mathcal{K} \mathcal{F}_{n}^{0}$ which admit a word representation of length $\leq 4$ are already contained in a subset listed in the table.

The sets $(F I K I)_{r},(F K I K)_{r}$, and $(F K I F)_{r}$. Elements of $(F I K I)_{r}$ have the form $f_{y} i_{z} k_{*} i_{*}$, where $y<z$ by Lemma 2.9 There are $\left(\begin{array}{l}n \\ 2\end{array}\right)$ many such pairs $(y, z)$, so (B) $\#(F I K I)_{r} \leq\left(\begin{array}{l}n \\ 2\end{array}\right)$. For (A), note that for any $x$, the word $k_{x} f_{y} i_{z} k_{*} i_{*}=$ $f_{x} f_{y} i_{z} k_{*} i_{*}$ either reduces to a $\leq 4$ letter word or else lies in $(K F I K I)_{r}$, so it lies in $\mathcal{X}$; while $i_{x} F I K I=\{0\} K I=\{0\} \subseteq \mathcal{X}$ as well. The arguments are similar for $(F K I K)_{r}$ and $(F K I F)_{r}$.

The sets $(K F I K)_{r},(K F K I)_{r}$, and $(K F I F)_{r}$. All elements of $(K F I K)_{r}$ have the form $k_{y} f_{z} i_{w} k_{*}$ where $y>z$ and $z \leq w$, which implies $(K F I K)_{r} \subseteq(K F I)_{r} k_{1}$ and therefore $\#(K F I K)_{r} \leq \#(K F I)_{r} \leq 2\left(\begin{array}{c}n+1 \\ 3\end{array}\right)$, establishing (B). For $1 \leq$ $x \leq n$, we have $k_{x} k_{y} f_{z} i_{w} k_{*}=k_{\max (x, y)} f_{z} i_{w} k_{*}$ by Lemma 2.3 and $i_{x} k_{y} f_{z} i_{w} k_{*}=$ $i_{x} k_{*} f_{z} i_{w} k_{*}=i_{x} f_{z} i_{w} k_{*}$ by Lemma 2.4. and $f_{x} k_{y} f_{z} i_{w} k_{*}=k_{\max (x, y)} f_{z} i_{w} k_{*}$ by Lemma 2.12 Each of these words has a representation of length $\leq 4$, and therefore lies in $\mathcal{X}$, establishing (A). The arguments are similar for $(K F K I)_{r}$ and $(K F I F)_{r}$.

The set $(K F K F)_{r}$. For $1 \leq x \leq n$, we have $k_{x} K F K F \subseteq K F K F \subseteq \mathcal{X}$ by Lemma 2.3 $i_{x} K F K F \subseteq I F K F \subseteq \mathcal{X}$ by Lemma2.4, and $f_{x} K F K F \subseteq K F K F \subseteq \mathcal{X}$ by Lemma 2.12 , so (A) holds.

To establish (B), we observe that every element of $(K F K F)_{r}$ has the form $k_{x} f_{y} k_{z} f_{w}$, and because this cannot be shortened to a word of length $\leq 3$, we must have $x>y$ by Lemma 2.3 and $y\langle z, z>w$ by Lemma 2.8. So we are looking for ordered quadruples $(x, y, z, w)$ which alternate in magnitude with $x>y, y<z$, $z>w$. There are $\left(\begin{array}{l}n \\ 2\end{array}\right)$ many such quadruples if $x=z$ and $y=w$; there are $2\left(\begin{array}{l}n \\ 3\end{array}\right)$ many if $x=z$ but $y \neq w$; and there are $2\left(\begin{array}{l}n \\ 3\end{array}\right)$ many if $y=w$ but $x \neq z$. If $x=w$, then necessarily $y<x$ and $z>x$, which yields an additional $\left(\begin{array}{l}n \\ 3\end{array}\right)$ possible quadruples. If all of $x, y, z, w$ are distinct, then either $x$ or $z$ is maximal. If $x$ is maximal then the choice of minimality for $y$ or $w$ determines the quadruple, yielding $2\left(\begin{array}{l}n \\ 4\end{array}\right)$ quadruples. If $z$ is maximal then either $y$ or $w$ is minimal; if $w$ is minimal the 
quadruple is determined, whereas if $y$ is minimal then there are 2 ways to assign $x$ and $w$. This gives another $(1+2)\left(\begin{array}{l}n \\ 4\end{array}\right)$ quadruples where $z$ is maximal. Thus we compute a bound of \# $(K F K F)_{r} \leq\left(\begin{array}{l}n \\ 2\end{array}\right)+(2+2+1)\left(\begin{array}{l}n \\ 3\end{array}\right)+(2+1+2)\left(\begin{array}{l}n \\ 4\end{array}\right)$, as in the table.

At this point, our computations up to this point have shown:

- All elements of $\mathcal{K F}_{n}^{0}$ which admit a word representation of length $\leq 5$ are already contained in a subset listed in the table.

The sets $(K F I K I)_{r},(K F K I K)_{r}$, and $(K F K I F)_{r}$. Every element of $(K F I K I)_{r}$ has the form $k_{y} f_{z} i_{w} k_{*} i_{*}$, and for any $1 \leq x \leq n$, we have $k_{x} k_{y} f_{z} i_{w} k_{*} i_{*}=$ $f_{x} k_{y} f_{z} i_{w} k_{*} i_{*}=k_{\max (x, y)} f_{z} i_{w} k_{*} i_{*}$ by Lemmas 2.3 and 2.12, while $i_{x} k_{y} f_{z} i_{w} k_{*} i_{*}=$ $i_{x} k_{*} f_{z} i_{w} k_{*} i_{*}=i_{x} f_{z} i_{w} k_{*} i_{*}$ by Lemma 2.4. In all three cases we find representations of length $\leq 5$, so $k_{x}(K F I K I)_{r}, i_{x}(K F I K I)_{r}, f_{x}(K F I K I)_{r} \subseteq \mathcal{X}$ and we have proven (A).

For (B), we note that since $k_{y} f_{z} i_{w} k_{*} i_{*}$ does not reduce to a word of length $\leq 4$, we must have $y>z$ by Lemma 2.3 , and by Lemma 2.9 we have $w>z$. Thus we are looking for triples $(y, z, w)$ with $y>z$ and $z<w$. By arguments analogous to those in the case of $(F K F)_{r}$, we compute that $\#(K F I K I)_{r} \leq\left(\begin{array}{l}n \\ 2\end{array}\right)+2\left(\begin{array}{l}n \\ 3\end{array}\right)$. The arguments for $(K F K I K)_{r}$ and $(K F K I F)_{r}$ are similar.

This completes the proof.

Example 4.2 (Separating KFKF Words). In [1], the authors show that $\# \mathcal{K}_{n} \leq$ $12 n+2$ for a saturated $n$-topological space, so we expect the size of the Kuratowski monoid to grow linearly with $n$. Our corresponding formula $p(n)$ in Theorem 1.1 implies quartic growth for the Kuratowski-Gaida-Eremenko monoid $\mathcal{K F}_{n}$. As is evident from the proof, the sole reason for this is that the set of reduced words $(K F K F)_{r}=\left\{k_{x} f_{y} k_{z} f_{w}: x>y, y<z, z>w, 1 \leq x, y, z, w \leq n\right\}$ is expected to contain $\left(\begin{array}{l}n \\ 2\end{array}\right)+5\left(\begin{array}{l}n \\ 3\end{array}\right)+5\left(\begin{array}{c}n \\ 4\end{array}\right)$ elements.

It is interesting to see a natural example of a saturated 4-topological space in which the elements of $(K F K F)_{r}$ are distinct. Consider $\left(\mathbb{R}^{3}, \tau_{1}, \tau_{2}, \tau_{3}, \tau_{4}\right)$, where $\tau_{1}=\tau_{s} \times \tau_{s} \times \tau_{s}, \tau_{2}=\tau_{s} \times \tau_{s} \times \tau_{u}, \tau_{3}=\tau_{s} \times \tau_{u} \times \tau_{u}$, and $\tau_{4}=\tau_{u} \times \tau_{u} \times \tau_{u}$. Define $B=((1,2) \times(0,2) \times(0,2)) \cup((0,2) \times(1,2) \times(0,2)) \cup((0,2) \times(0,2) \times(1,2))$, and let $\left\{C_{n}: n \in \mathbb{N}\right\}$ be a countably infinite collection of pairwise disjoint $\tau_{4}$-closed sub-cubes of $(0,1) \times(0,1) \times(0,1)$ with the property that if $C=\bigcup_{n \in \mathbb{N}} C_{n}$, then the set of $\tau_{4}$-derived points of $C$ is exactly $C^{\prime}=k_{4} C \backslash C=(\{1\} \times[0,1] \times[0,1]) \cup$ $([0,1] \times\{1\} \times[0,1]) \cup([0,1] \times[0,1] \times\{1\})$. We denote $B_{\mathbb{Q}}=B \cap(\mathbb{Q} \times \mathbb{Q} \times \mathbb{Q})$, and we take for our initial set $A=B_{\mathbb{Q}} \cup\left(\bigcup_{n \in \mathbb{N}} i_{4} C\right)$.

We also consider the particular open cube $i_{4} C_{0} \subseteq A$, say $i_{4} C_{0}=\left(x_{0}, x_{1}\right) \times$ $\left(y_{0}, y_{1}\right) \times\left(z_{0}, z_{1}\right)$, and we label the following sets:

$$
\begin{aligned}
\phi & =\left(x_{0}, x_{1}\right) \times\left(y_{0}, y_{1}\right) \times\left\{z_{1}\right\} \\
\psi & =\left(x_{0}, x_{1}\right) \times\left\{y_{1}\right\} \times\left(z_{0}, z_{1}\right) \\
q & =(0,1) \times\{1\} \times(0,1) \\
r & =\{1\} \times(0,1) \times(0,1) \\
Q & =(0,2) \times\{2\} \times(0,2)
\end{aligned}
$$

$=$ the upper face of $C_{0}$;

$=$ the forward face of $C_{0}$;

$=$ the inner rear face of $B$;

$=$ the inner left face of $B$;

$=$ the forward face of $B$; 
$R=\{2\} \times(0,2) \times(0,2)$

$U=[((1,2) \times(0,2)) \cup((0,2) \times(1,2))] \times\{0\}$

$V=((1,2) \times\{0\} \times(0,2)) \cup((0,2) \times\{0\} \times(1,2))$
$=$ the right face of $B$; $=$ the outer lower face of $B$; $=$ the outer rear face of $B$.

Then by direct computation, one may verify the following properties about the sets $k_{x} f_{y} k_{z} f_{w} A$, which differentiate all possible ordered quadruples $(x, y, z, w)$ satisfying $x>y, y<z, z>w$ :

(1) (a) If $w=1$ then $\phi, \psi$ are disjoint from $k_{x} f_{y} k_{z} f_{w} A$.

(b) If $w=2$ then $\phi \subseteq k_{x} f_{y} k_{z} f_{w} A$ but $\psi \cap k_{x} f_{y} k_{z} f_{w} A=\emptyset$.

(c) If $w=3$ then $\phi, \psi \subseteq k_{x} f_{y} k_{z} f_{w} A$.

(2) (a) If $z=2$ then $Q, R$ are disjoint from $k_{x} f_{y} k_{z} f_{w} A$.

(b) If $z=3$ then $Q \subseteq k_{x} f_{y} k_{z} f_{w} A$ but $R \cap k_{x} f_{y} k_{z} f_{w} A=\emptyset$.

(c) If $z=4$ then $Q, R \subseteq k_{x} f_{y} k_{z} f_{w} A$.

(3) (a) If $y=1$ then $U, V$ are disjoint from $k_{x} f_{y} k_{z} f_{w} A$.

(b) If $y=2$ then $U \subseteq k_{x} f_{y} k_{z} f_{w} A$ but $V \cap k_{x} f_{y} k_{z} f_{w}=\emptyset$.

(c) If $y=3$ then $U, V \subseteq k_{x} f_{y} k_{z} f_{w} A$.

(4) (a) If $x=2$ then $q, r$ are disjoint from $k_{x} f_{y} k_{z} f_{w} A$.

(b) If $x=3$ then $q \subseteq k_{x} f_{y} k_{z} f_{w} A$ but $r \cap k_{x} f_{y} k_{z} f_{w} A=\emptyset$.

(c) If $x=4$ then $q, r \subseteq k_{x} f_{y} k_{z} f_{w} A$.

From the above, distinct quadruples $(x, y, z, w)$ yield distinct sets $k_{x} f_{y} k_{z} f_{w} A$, and therefore

Figure 3. From left to right: the set $C_{0}$ and its faces; the set $B$ and its faces; typical basic open neighborhoods in $\tau_{1}, \tau_{2}, \tau_{3}$.

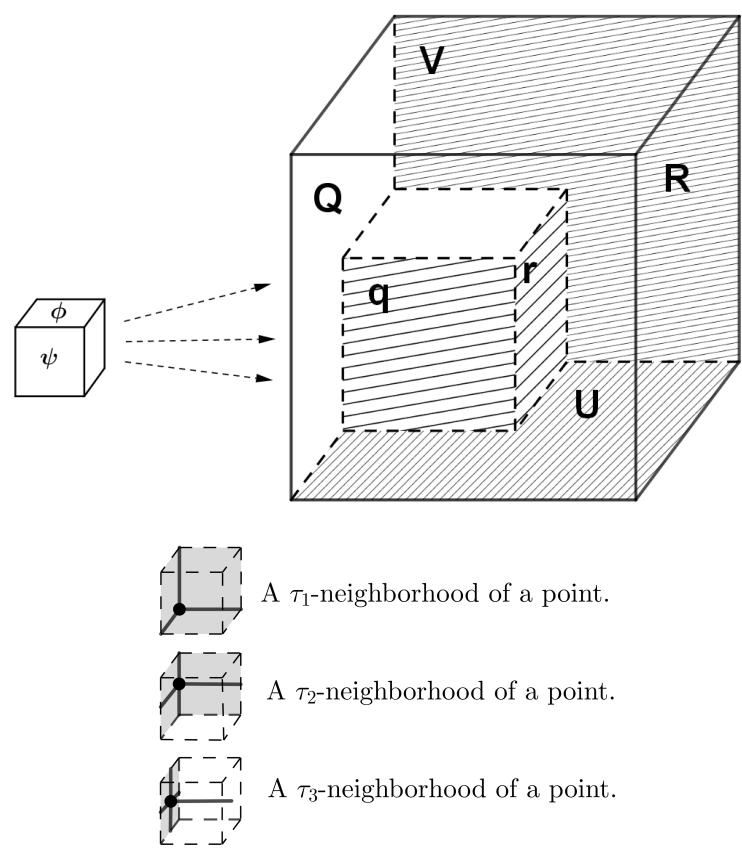




$$
\begin{gathered}
\#(K F K F)_{r}[A]=\#\left\{k_{x} f_{y} k_{z} f_{w} A: 1 \leq x, y, z \leq n, x>y, y<z, z>w\right\}= \\
\left(\begin{array}{l}
4 \\
2
\end{array}\right)+5\left(\begin{array}{c}
4 \\
3
\end{array}\right)+5\left(\begin{array}{c}
4 \\
2
\end{array}\right)=31 .
\end{gathered}
$$

\section{Separating Kuratowski-Gaida-Eremenko Words}

The goal of this section is to prove that our upper bound $p(n)$ is sharp for every $n$. Guided by the results of the previous section, we introduce the following definition: a word in the generators $\left\{k_{x}, i_{x}, f_{x}: 1 \leq x \leq n\right\}$ (formally, an element of the free semigroup on $3 n$ letters) will be called a Kuratowski-Gaida-Eremenko word, or KGE-word, if it has one of the following forms:

- Id or $0=i_{*} f_{*} k_{*}$,

- $k_{x}, i_{x}, i_{x} k_{*}, k_{x} i_{*}, i_{x} k_{*} i_{*}, k_{x} i_{*} k_{*}, f_{x}, i_{x} f_{*}$, or $k_{x} i_{*} f_{*}$,

- $f_{x} f_{y}$,

- $k_{x} f_{y}$ where $x>y$,

- $f_{x} i_{y} k_{*} i_{*}, f_{x} k_{y} i_{*} k_{*}$, or $f_{x} k_{y} i_{*} f_{*}$ where $x<y$,

- $f_{x} i_{y}, f_{x} k_{y}, f_{x} i_{y} f_{*}, f_{x} i_{y} k_{*}$, or $f_{x} k_{y} i_{*}$ where $x \leq y$,

- $k_{x} f_{y} f_{z}$ where $x>y$,

- $f_{x} k_{y} f_{z}$ where $x<y$ and $y<z$,

- $k_{x} f_{y} k_{z}, k_{x} f_{y} i_{z}, k_{x} f_{y} i_{z} k_{*}, k_{x} f_{y} k_{z} i_{*}$, or $k_{x} f_{y} i_{z} f_{*}$ where $x>y$ and $y \leq z$,

- $k_{x} f_{y} i_{z} k_{*} i_{*}, k_{x} f_{y} k_{z} i_{*} k_{*}$, or $k_{x} f_{y} k_{z} i_{*} f_{*}$ where $x>y$ and $y<z$,

- $k_{x} f_{y} k_{z} f_{w}$ where $x>y, y<z$, and $z>w$.

We understand the $*$-notation as imposing an equivalence relation on the KGEwords: for example, although strictly speaking $i_{1} f_{1} k_{1}$ and $i_{1} f_{1} k_{2}$ are distinct words in the free semigroup, we regard them here as merely two representations of the same KGE-word 0; on the other hand $f_{1} f_{1}$ and $f_{1} f_{2}$ are distinct KGE-words. With this understanding in place, the number of KGE-words is $p(n)$. For convenience, we allow words and operators to be used interchangeably when the precise meaning is clear. So each KGE-word corresponds to at most one element of $\mathcal{K} \mathcal{F}_{n}$, whereas a priori an element of $\mathcal{K} \mathcal{F}_{n}$ may be represented by more than one KGE-word.

We note that in any monoid $\mathcal{K F}_{n}^{0}$, by Lemmas 2.3 through 2.12 , we have the following set inclusions:

$$
\begin{aligned}
& K F \supseteq(K F)_{r} \cup F ; \\
& K F K \supseteq(K F K)_{r} \cup F K ; \\
& K F I \supseteq(K F I)_{r} \cup F I ; \\
& K F I F \supseteq(K F I F)_{r} \cup F I F ; \\
& K F K I F \supseteq(K F K I F)_{r} \cup(F K I F)_{r} ; \\
& K F I K \supseteq(K F I K)_{r} \cup F I K ; \\
& K F K I K \supseteq(K F K I K)_{r} \cup(F K I K)_{r} ; \\
& K F K I \supseteq(K F K I)_{r} \cup F K I ; \\
& K F I K I \supseteq(K F I K I)_{r} \cup(F I K I)_{r} ; \\
& K F K F \supseteq(K F K F)_{r} \cup(K F F)_{r} \cup(F K F)_{r} \cup F F .
\end{aligned}
$$

Therefore the following holds. 
Proposition 5.1. Each KGE-word belongs to at least one of the following sets in $\mathcal{K F}_{n}^{0}$ :

- $\{\operatorname{Id}\}$ or $\{0\}$;

- $K, I, I K, K I, K I K, I K I, I F, K I F$;

- $K F, K F K, K F I$;

- $K F K F$; or

- KFIF $\cup K F K I F, K F I K \cup K F K I K, K F K I \cup K F I K I$.

For the reader's convenience, we note that the 17 sets above correspond to the 17 distinct even operators which comprise the monoid $\mathcal{K} \mathcal{F}_{1}^{0}$ from $\mathbf{5}$.

Theorem 1.2. For every $n \geq 1$, there exists a saturated polytopological space $\left(X, \tau_{1}, \ldots, \tau_{n}\right)$ in which $\# \mathcal{K F}_{n}^{0}=p(n)$ and $\# \mathcal{K F}_{n}=2 p(n)$. In fact, there is an initial set $A \subseteq X$ such that $\#\left\{o A: o \in \mathcal{K F}_{n}\right\}=2 p(n)$.

Proof. Applying Lemma 3.2 , it suffices to demonstrate the following: For any pair of distinct KGE-words $\omega_{1}, \omega_{2} \in \mathcal{K} \mathcal{F}_{n}$, there exists a saturated $n$-topological space $X^{\omega_{1}, \omega_{2}}$ and a subset $A^{\omega_{1}, \omega_{2}} \subseteq X^{\omega_{1}, \omega_{2}}$ in which $\omega_{1} A^{\omega_{1}, \omega_{2}} \neq \omega_{2} A^{\omega_{1}, \omega_{2}}$. We verify the claim for $\omega_{1} \neq \omega_{2}$ by using the cases delineated in Proposition 5.1 .

Case 1: $\omega_{1} \in E_{1}$ and $\omega_{2} \in E_{2}$, where $E_{1}$ and $E_{2}$ are distinct subsets from Proposition 5.1. Then we may take for our separating space $\left(\mathbb{R}, \tau_{1}, \ldots, \tau_{n}\right)$ where $\tau_{1}=\ldots=\tau_{n}=$ $\overline{\tau_{u}}$, and take for our initial set $A$ the example exhibited by Gaida-Eremenko in [5. In this case, because all topologies are equal, the monoid $\mathcal{K F}_{n}^{0}$ is actually equal to $\mathcal{K F}_{1}^{0}$ and we get the following reductions: $K F I F \cup K F K I F=F I F$, $K F I K \cup K F K I K=F I K, K F K I \cup K F I K I=F K I, K F K F=F F, K F=F$, $K F K=F K, K F I=F I$. But elements $\omega_{1}, \omega_{2}$ taken from distinct word types will produce different sets $\omega_{1} A \neq \omega_{2} A$, as demonstrated by Gaida and Eremenko.

Case 2: $\omega_{1}, \omega_{2} \in E$ where $E=K, I, I K, K I, K I K, I K I, I F$, or $K I F$. Assume that, for example, that $\omega_{1}, \omega_{2} \in K I K$. We have $\omega_{1}=k_{x_{1}} i_{*} k_{*}$ and $\omega_{2}=k_{x_{2}} i_{*} k_{*}$ where $1 \leq x_{1}, x_{2} \leq n$, and since $\omega_{1} \neq \omega_{2}$, we have $x_{1} \neq x_{2}$. Assume without loss of generality that $x_{1}<x_{2}$, and take for a separating space $\left(\mathbb{R}, \tau_{1}, \ldots, \tau_{n}\right)$ where $\tau_{1}=\ldots=\tau_{x_{1}}=\tau_{s}$ and $\tau_{x_{1}+1}=\ldots=\tau_{n}=\tau_{u}$. Take the initial set $A$ from Example 3.3 Then $\omega_{1} A=k_{1} i_{*} k_{*} A \neq k_{n} i_{*} k_{*} A=\omega_{2} A$. The proofs for the other sets $E=K, I, \ldots$ etc. are similar because words in these sets $E$ depend on only one index, and we leave them to the reader.

Case 3: $\omega_{1}, \omega_{2} \in K F$. If $\omega_{1}, \omega_{2} \in K F$, then we have $\omega_{1}=k_{x_{1}} f_{y_{1}}$ and $\omega_{2}=k_{x_{2}} f_{y_{2}}$ where $1 \leq x_{1}, y_{1}, x_{2}, y_{2} \leq n, x_{1} \geq y_{1}$, and $x_{2} \geq y_{2}$. Assuming $\left(x_{1}, y_{1}\right) \neq\left(x_{2}, y_{2}\right)$, we have either $x_{1} \neq x_{2}$ or $y_{1} \neq y_{2}$.

Sub-Case (a): Suppose $y_{1} \neq y_{2}$; without loss of generality assume $y_{1}<y_{2}$. Then take for a separating space $\left(\mathbb{R}, \tau_{1}, . ., \tau_{n}\right)$ where $\tau_{1}=\ldots=\tau_{y_{1}}=\tau_{s}$ and $\tau_{y_{1}+1}=\ldots=\tau_{n}=\tau_{u}$, and take for an initial set $A$ as in Example 3.3. Then we have $\omega_{1}=k_{x_{1}} f_{y_{1}}=k_{x_{1}} f_{1}$, which is equal to either $f_{1}$ or $k_{n} f_{1}$ depending on the value of $x_{1}$. On the other hand since $x_{2} \geq y_{2}>y_{1}$, we have $\omega_{2}=k_{x_{2}} f_{y_{2}}=k_{n} f_{n}=f_{n}$. Since $f_{1} A \neq k_{n} f_{1} A \neq f_{n} A$, we conclude $\omega_{1} A \neq \omega_{2} A$ as desired. 
Sub-Case (b): Suppose $y_{1}=y_{2}$ but $x_{1} \neq x_{2}$; without loss of generality assume $x_{1}<x_{2}$. Take for a separating space $\left(\mathbb{R}, \tau_{1}, . ., \tau_{n}\right)$ where $\tau_{1}=\ldots=\tau_{x_{1}}=\tau_{s}$ and $\tau_{x_{1}+1}=\ldots=\tau_{n}=\tau_{u}$, and take the usual initial set $A$ as in Example 3.3. Then since $y_{1} \leq x_{1}$, we have $\omega_{1}=k_{x_{1}} f_{y_{1}}=k_{1} f_{1}=f_{1}$, while since $y_{2}=y_{1} \leq x_{1}$, we have $\omega_{2}=k_{x_{2}} f_{y_{2}}=k_{n} f_{1}$. So $\omega_{1} A \neq \omega_{2} A$ as in Example 3.3 .

Case 4: $\omega_{1}, \omega_{2} \in K F K$. The idea of this proof is the same as in Case 3. Suppose that $\omega_{1}, \omega_{2} \in K F K$, then we have $\omega_{1}=k_{x_{1}} f_{y_{1}} k_{z_{1}}$ and $\omega_{2}=k_{x_{2}} f_{y_{2}} k_{z_{2}}$ where $1 \leq x_{1}, y_{1}, z_{1}, x_{2}, y_{2}, z_{2} \leq n, x_{1} \geq y_{1}, y_{1} \leq z_{1}, x_{2} \geq y_{2}, y_{2} \leq z_{2}$. We have $\left(x_{1}, y_{1}, z_{1}\right) \neq\left(x_{2}, y_{2}, z_{2}\right)$, and therefore $x_{1} \neq x_{2}, y_{1} \neq y_{2}$ or $z_{1} \neq z_{2}$.

Sub-Case (a): Suppose $z_{1} \neq z_{2}$, so without loss of generality $z_{1}<z_{2}$. Take for a separating space $\left(\mathbb{R}, \tau_{1}, . ., \tau_{n}\right)$ where $\tau_{1}=\ldots=\tau_{z_{1}}=\tau_{s}$ and $\tau_{z_{1}+1}=\ldots=\tau_{n}=\tau_{u}$, and take for an initial set $A$ as in Example 3.3. Then since $y_{1} \leq z_{1}$, we have $\omega_{1}=k_{x_{1}} f_{y_{1}} k_{z_{1}}=k_{x_{1}} f_{1} k_{1}$, which is equal to either $f_{1} k_{1}$ or $k_{n} f_{1} k_{1}$ depending on the value of $x_{1}$. On the other hand $\omega_{2}=k_{x_{2}} f_{y_{2}} k_{n}$, so $\omega_{2}$ is equal to either $k_{1} f_{1} k_{n}=f_{1} k_{n}, k_{n} f_{1} k_{n}$, or $k_{n} f_{n} k_{n}=f_{n} k_{n}$, depending on the values of $x_{2}, y_{2}$. These five distinct possibilities yield five distinct sets when applied to $A$, so we conclude $\omega_{1} A \neq \omega_{2} A$ as desired.

Sub-Case (b): Suppose $z_{1}=z_{2}$ but $y_{1}<y_{2}$. Take for a separating space $\left(\mathbb{R}, \tau_{1}, . ., \tau_{n}\right)$ where $\tau_{1}=\ldots=\tau_{y_{1}}=\tau_{s}$ and $\tau_{y_{1}+1}=\ldots=\tau_{n}=\tau_{u}$, and take the usual initial set $A$ as in Example 3.3 . Then, considering all possible values of $x_{1}, z_{1}$, we compute that $\omega_{1}=k_{x_{1}} f_{1} k_{z_{1}} \in\left\{f_{1} k_{1}, f_{1} k_{n}, k_{n} f_{1} k_{1}, k_{n} f_{1} k_{n}\right\}$. On the other hand since $x_{2}, z_{2} \geq y_{2}>y_{1}$, we have $\omega_{2}=k_{n} f_{n} k_{n}=f_{n} k_{n}$. So $\omega_{1} A \neq \omega_{2} A$.

Sub-Case (c): Suppose $z_{1}=z_{2}$ and $y_{1}=y_{2}$ but $x_{1}<x_{2}$. Take for a separating space $\left(\mathbb{R}, \tau_{1}, . ., \tau_{n}\right)$ where $\tau_{1}=\ldots=\tau_{x_{1}}=\tau_{s}$ and $\tau_{x_{1}+1}=\ldots=\tau_{n}=\tau_{u}$, and take the usual initial set $A$ as in Example 3.3 . We compute $\omega_{1}=k_{x_{1}} f_{y_{1}} k_{z_{1}}=$ $k_{1} f_{1} k_{z_{1}} \in\left\{f_{1} k_{1}, f_{1} k_{n}\right\}$, and $\omega_{2}=k_{x_{2}} f_{y_{2}} k_{z_{2}}=k_{n} f_{y_{2}} k_{z_{2}} \in\left\{k_{n} f_{1} k_{1}, k_{n} f_{1} k_{n}, f_{n} k_{n}\right\}$, so $\omega_{1} A \neq \omega_{2} A$.

Case 5: $\omega_{1}, \omega_{2} \in K F I$. In this case take the same separating space as in Case 4, but for an initial set take $c A$ where $A$ is the initial set from Case 4 . We are done if $\omega_{1} c A \neq \omega_{2} c A$, and this follows from Case 4 because both $\omega_{1} c$ and $\omega_{2} c$ are elements of $K F K$. (To verify this, write $\omega_{1}=k_{x_{1}} f_{y_{1}} i_{z_{1}}$ where $1 \leq x_{1}, y_{1}, z_{1} \leq n, x_{1} \geq y_{1}$, and $y_{1} \leq z_{1}$. Then $\omega_{1} c=k_{x_{1}} f_{y_{1}} c k_{z_{1}}=k_{x_{1}} f_{y_{1}} k_{z_{1}} \in K F K$, and similarly for $\omega_{2}$.)

Case 6: $\omega_{1}, \omega_{2} \in K F K F$. We proceed similarly to Cases 3 and 4 . We have $\omega_{1}=$ $k_{x_{1}} f_{y_{1}} k_{z_{1}} f_{w_{1}}$ and $\omega_{2}=k_{x_{2}} f_{y_{2}} k_{z_{2}} f_{w_{2}}$ where $1 \leq x_{1}, y_{1}, z_{1}, w_{1}, x_{2}, y_{2}, z_{2}, w_{2} \leq n$, $x_{1} \geq y_{1}, y_{1} \leq z_{1}, z_{1} \geq w_{1}, x_{2} \geq y_{2}, y_{2} \leq z_{2}$, and $z_{2} \geq w_{2}$. We also know $\left(x_{1}, y_{1}, z_{1}, w_{1}\right) \neq\left(x_{2}, y_{2}, z_{2}, w_{2}\right)$, which gives us four sub-cases.

Sub-Case (a): Suppose $w_{1} \neq w_{2}$, so without loss of generality $w_{1}<w_{2}$. We consider $\left(\mathbb{R}, \tau_{1}, \ldots, \tau_{n}\right)$ with $\tau_{1}=\ldots=\tau_{w_{1}}=\tau_{s}$ and $\tau_{w_{1}+1}=\ldots=\tau_{n}=\tau_{u}$. Considering all possible values of $x_{1}, y_{1}, z_{1}, x_{2}, y_{2}, z_{2}$, we compute that

$$
\begin{aligned}
& \omega_{1}=k_{x_{1}} f_{y_{1}} k_{z_{1}} f_{1} \in\left\{f_{1} f_{1}, f_{n} f_{1}, f_{1} k_{n} f_{1}, k_{n} f_{1} f_{1}, k_{n} f_{1} k_{n} f_{1}\right\} \\
& \omega_{2}=k_{x_{2}} f_{y_{2}} k_{z_{2}} f_{n} \in\left\{f_{1} f_{n}, f_{n} f_{n}, k_{n} f_{1} f_{n}\right\}
\end{aligned}
$$


from which we conclude $\omega_{1} A \neq \omega_{2} A$, where $A$ is the initial set from Example 3.3 .

Sub-Case (b): Suppose $w_{1}=w_{2}$ but $z_{1}<z_{2}$, and consider $\left(\mathbb{R}, \tau_{1}, \ldots, \tau_{n}\right)$ where $\tau_{1}=\ldots=\tau_{z_{1}}=\tau_{s}$ and $\tau_{z_{1}+1}=\ldots=\tau_{n}=\tau_{u}$. Since $w_{1}, y_{1} \leq z_{1}$, we get $\omega_{1}=$ $k_{x_{1}} f_{1} k_{1} f_{1} \in\left\{f_{1} f_{1}, k_{n} f_{1} f_{1}\right\}$ whereas $\omega_{2}=k_{x_{2}} f_{y_{2}} k_{n} f_{1} \in\left\{f_{1} k_{n} f_{1}, k_{n} f_{1} k_{n} f_{1}, f_{n} f_{1}\right\}$, so $\omega_{1} A \neq \omega_{2} A$ where $A$ is as in Example 3.3 .

Sub-Case (c): Suppose now $w_{1}=w_{2}, z_{1}=z_{2}$ but $y_{1}<y_{2}$, and consider $\left(\mathbb{R}, \tau_{1}, \ldots, \tau_{n}\right)$ where $\tau_{1}=\ldots=\tau_{y_{1}}=\tau_{s}$ and $\tau_{y_{1}+1}=\ldots=\tau_{n}=\tau_{u}$. Since $z_{1}=$ $z_{2} \geq y_{2}$, we get $\omega_{1}=k_{x_{1}} f_{1} k_{n} f_{w_{1}} \in\left\{f_{1} k_{n} f_{1}, k_{n} f_{1} k_{n} f_{1}, f_{1} f_{n}, k_{n} f_{1} f_{n}\right\}$, whereas since $x_{2}, z_{2} \geq y_{2}$, we have $\omega_{2}=k_{n} f_{n} k_{n} f_{w_{2}} \in\left\{f_{n} f_{1}, f_{n} f_{n}\right\}$, so $\omega_{1} A \neq \omega_{2} A$ where $A$ is as in Example 3.3 .

Sub-Case (d): Suppose $w_{1}=w_{2}, z_{1}=z_{2}, y_{1}=y_{2}$ but $x_{1}<x_{2}$, and consider $\left(\mathbb{R}, \tau_{1}, \ldots, \tau_{n}\right)$ where $\tau_{1}=\ldots=\tau_{x_{1}}=\tau_{s}$ and $\tau_{x_{1}+1}=\ldots=\tau_{n}=\tau_{u}$. Since $y_{1} \leq$ $x_{1}$, we get $\omega_{1}=k_{1} f_{1} k_{z_{1}} f_{w_{1}} \in\left\{f_{1} f_{1}, f_{1} f_{n}, f_{1} k_{n} f_{1}\right\}$ whereas $\omega_{2}=k_{n} f_{y_{2}} k_{z_{2}} f_{w_{2}} \in$ $\left\{k_{n} f_{1} f_{1}, k_{n} f_{1} f_{n}, k_{n} f_{1} k_{n} f_{1}, f_{n} f_{1}\right\}$, so $\omega_{1} A \neq \omega_{2} A$ where $A$ is as in Example 3.3 .

Case 7: $\omega_{1}, \omega_{2} \in K F I F \cup K F K I F$. We proceed similarly to Cases 3 , 4, and 6 . Observe that we may write

$$
\omega_{1}=k_{x_{1}} f_{y_{1}} \sigma_{z_{1}} i_{1} f_{*}
$$

with $x_{1} \geq y_{1}, y_{1} \leq z_{1}$, where either $\sigma_{z_{1}}=i_{z_{1}} \in I$ (in case $\omega_{1} \in$ KFIF) or $\sigma_{z_{1}}=k_{z_{1}} \in K$ where $z_{1}>y_{1}$ (in case $\omega_{1} \in K F K I F \backslash K F I F$ ). Similarly, we may write $\omega_{2}$ as

$$
\omega_{2}=k_{x_{2}} f_{y_{2}} \rho_{z_{2}} i_{1} f_{*}
$$

where $x_{2} \geq y_{2}, y_{2} \leq z_{2}$, and either $\rho_{z_{2}}=i_{z_{2}}$ or else $\rho_{z_{2}}=k_{z_{2}}$ and $z_{2}>y_{2}$. Since $\omega_{1} \neq \omega_{2}$, there are four sub-cases: either $z_{1} \neq z_{2}$; or $z_{1}=z_{2}$ but $\sigma_{z_{1}} \neq \rho_{z_{2}}$; or $y_{1} \neq y_{2}$; or $x_{1} \neq x_{2}$. In each of the four sub-cases below, we denote $\sigma_{1}=i_{1}$ and $\sigma_{n}=i_{n}$ if $\sigma_{z_{1}}=i_{z_{1}}$; and $\sigma_{1}=k_{1}$ and $\sigma_{n}=k_{n}$ if $\sigma_{z_{1}}=k_{z_{1}}$. Similarly we allow $\rho_{1}, \rho_{n}$ to denote either $i_{1}, i_{n}$ or $k_{1}, k_{n}$ respectively as implied by the value of $\rho_{z_{2}}$.

Sub-Case (a): Suppose $z_{1}<z_{2}$. Consider the separating space $\left(\mathbb{R}, \tau_{1}, \ldots, \tau_{n}\right)$ where $\tau_{1}=\ldots=\tau_{z_{1}}=\tau_{s}$ and $\tau_{z_{1}+1}=\ldots=\tau_{n}=\tau_{u}$. Since $y_{1} \leq z_{1}$, we have $\omega_{1}=k_{x_{1}} f_{1} \sigma_{1} i_{1} f_{*}=k_{x_{1}} f_{1} i_{1} f_{*}$, so $\omega_{1}=f_{1} i_{1} f_{*}$ or $\omega_{1}=k_{n} f_{1} i_{1} f_{*}$, depending on the value of $x_{1}$. On the other hand, considering all possible values of $x_{2}, y_{2}$, and $\rho_{z_{2}}=\rho_{n}$, we compute

$$
\omega_{2}=k_{x_{2}} f_{y_{2}} \rho_{n} i_{1} f_{*} \in\left\{f_{1} i_{n} f_{*}, f_{1} k_{n} i_{*} f_{*}, f_{n} i_{n} f_{*}, k_{n} f_{1} k_{n} i_{*} f_{*}, k_{n} f_{1} i_{n} f_{*}\right\} .
$$

It follows that $\omega_{1} A \neq \omega_{2} A$, where $A$ is the initial set from Example 3.3 .

Sub-Case (b): Suppose $z_{1}=z_{2}$, but $\sigma_{z_{1}}=k_{z_{1}}$ with $z_{1}>y_{1}$, while $\rho_{z_{2}}=i_{z_{2}}$. We take the separating space $\left(\mathbb{R}, \tau_{1}, \ldots, \tau_{n}\right)$ where $\tau_{1}=\ldots=\tau_{y_{1}}=\tau_{s}$ and $\tau_{y_{1}+1}=$ $\ldots=\tau_{n}=\tau_{u}$. We have $\omega_{1}=k_{x_{1}} f_{1} k_{n} i_{1} f_{*} \in\left\{f_{1} k_{n} i_{*} f_{*}, k_{n} f_{1} k_{n} i_{*} f_{*}\right\}$, while since $z_{2}=z_{1}>y_{1}$, we have $\omega_{2}=k_{x_{2}} f_{y_{2}} i_{n} i_{1} f_{*}=k_{x_{2}} f_{y_{2}} i_{n} f_{*} \in\left\{f_{1} i_{n} f_{*}, f_{n} i_{n} f_{*}, k_{n} f_{1} i_{n} f_{*}\right\}$. So $\omega_{1} A \neq \omega_{2} A$, taking $A$ from Example 3.3 .

Sub-Case (c): Suppose $y_{1}<y_{2}$, and take the separating space $\left(\mathbb{R}, \tau_{1}, \ldots, \tau_{n}\right)$ where $\tau_{1}=\ldots=\tau_{y_{1}}=\tau_{s}$ and $\tau_{y_{1}+1}=\ldots=\tau_{n}=\tau_{u}$. We have

$$
\omega_{1}=k_{x_{1}} f_{1} \sigma_{z_{1}} i_{1} f_{*} \in\left\{f_{1} i_{1} f_{*}, f_{1} i_{n} f_{*}, f_{1} k_{n} i_{*} f_{*}, k_{n} f_{1} i_{1} f_{*}, k_{n} f_{1} i_{n} f_{*}, k_{n} f_{1} k_{n} i_{*} f_{*}\right\},
$$


whereas since $z_{2} \geq y_{2}$, we have $\omega_{2}=k_{x_{2}} f_{n} \rho_{n} i_{1} f_{*}=k_{x_{2}} f_{n} i_{n} f_{*}=f_{n} i_{n} f_{*}$. So $\omega_{1} A \neq \omega_{2} A$, taking $A$ from Example 3.3 .

Sub-Case (d): Suppose $y_{1}=y_{2}$, but $x_{1}<x_{2}$, and take the separating space $\left(\mathbb{R}, \tau_{1}, \ldots, \tau_{n}\right)$ where $\tau_{1}=\ldots=\tau_{x_{1}}=\tau_{s}$ and $\tau_{x_{1}+1}=\ldots=\tau_{n}=\tau_{u}$ with the initial set $A$ from Example 3.3. Since $y_{1}=y_{2} \leq x_{1}$, we have $\omega_{1}=k_{1} f_{1} \sigma_{z_{1}} i_{1} f_{*} \in$ $\left\{f_{1} i_{1} f_{*}, f_{1} i_{n} f_{*}, f_{1} k_{n} i_{*} f_{*}\right\}$ and $\omega_{2}=k_{n} f_{1} \rho_{z_{2}} i_{1} f_{*} \in\left\{k_{n} f_{1} i_{1} f_{*}, k_{n} f_{1} i_{n} f_{*}, k_{n} f_{1} k_{n} i_{*} f_{*}\right\}$, so $\omega_{1} A \neq \omega_{2} A$.

Case 8: $\omega_{1}, \omega_{2} \in K F I K \cup K F K I K$. In this case take the same separating space as in Case 7, but for an initial set take $f_{n} A$ where $A$ is the initial set from Case 7. We are done if $\omega_{1} f_{n} A \neq \omega_{2} f_{n} A$; but this follows from Case 7 because $\omega_{1} f_{n}, \omega_{2} f_{n} \in K F I F \cup K F K I F$.

Case 9: $\omega_{1}, \omega_{2} \in K F K I \cup K F I K I$. Take the same separating space as in Cases 7 and 8 , and for an initial set take $c A$ where $A$ is the initial set from Case 8. Then since $\omega_{1} c, \omega_{2} c \in K F I K \cup K F K I K$, we have $\omega_{1} c A \neq \omega_{2} c A$ by Case 8 .

\section{Acknowledgement}

We extend our sincere thanks to Mark Bowron for his comments and corrections.

\section{References}

[1] T. Banakh, O. Chervak, T. Martynyuk, M. Pylypovych, A. Ravsky, and M. Simkiv, Kuratowski monoids of $n$-topological spaces, Topol. Algebra Appl. 6 (2018), 1-25.

[2] E. Buchman, Problem E 3144, Amer. Math. Monthly 93 (1986), 299.

[3] M. Bowron, Kuratowski's Closure-Complement Cornucopia (2012), https:// mathtransit.com/cornucopia.php, retrieved on December 6, 2020.

[4] A. É. Eremenko, Diagram of the partial ordering of the closure-complementfrontier monoid, https://www.math.purdue.edu/ eremenko/dvi/table1. pdf, retrieved on January 2, 2020.

[5] Yu. R. Gaida and A. É. Eremenko, On the frontier operator in Boolean algebras with a closure, Ukr. Math. J. 26 (1974), 662-664.

[6] B. J. Gardner and M. Jackson, The Kuratowski closure-complement theorem, N. Z. J. Math. 38 (2008), 9-44.

[7] H. H. Herda and R. C. Metzler, Closure and interior in finite topological spaces, Colloq. Math. 15 (1966), 211--216.

[8] K. Kuratowski, Sur l'operation A de l'Analysis Situs, Fundamenta Mathematicae 3 (1922), 182--199.

[9] J. C. C. McKinsey and A. Tarski, The algebra of topology, Ann. Math. 45 (1944), $141-191$.

[10] J. Shallit and R. Willard, Kuratowski's Theorem for Two Closure Operators, ArXiv:1109.1227, 2011.

[11] D. Sherman, Variations on Kuratowski's 14-set theorem, Am. Math. Mon. 117 (2) (2010), 113-123. 
Sara Canilang

Department of Mathematics and Statistics,

Carleton College,

Northfield, MN 55057

sara.canilang@gmail.com

Nicolas Graese

Department of Mathematics and Statistics,

Carleton College,

Northfield, MN 55057

graesenah@gmail.com
Michael P. Cohen

Department of Mathematics and

Statistics,

Carleton College,

Northfield, MN 55057

mikepatrickcohen@gmail.com

Ian Seong

Department of Mathematics and

Statistics,

Carleton College,

Northfield, MN 55057

iseong@wisc.edu 